TRANSACTIONS OF THE

AMERICAN MATHEMATICAL SOCIETY

Volume 177, March 1973

\title{
ORIENTED AND WEAKLY COMPLEX BORDISM ALGEBRA OF FREE PERIODIC MAPS
}

BY

\section{KATSUYUKI SHIBATA}

\begin{abstract}
Free cyclic actions on a closed oriented (weakly almost complex, respectively) manifold which preserve the orientation (weakly complex structure) are considered from the viewpoint of equivariant bordism theory. The author gives an explicit presentation of the oriented bordism module structure and multiplicative structure of all orientation preserving (and reversing) free involutions. The odd period and weakly complex cases are also determined with the aid of the notion of formal group laws. These results are applied to a nonexistence problem for certain equivariant maps.
\end{abstract}

Introduction. As the oriented analogue of the free equivariant unoriented bordism theory $\hat{\Re}_{*}(X, A, \tau)$ of Stong [12], K. Komiya and C. $\mathrm{M}$. Wu have respectively defined the free equivariant oriented bordism theories $\hat{\Omega}_{*}^{+}(X, A, \tau)$ and $\hat{\Omega}_{*}^{-}(X, A, \tau)$ for involutions $(X, A, \tau)$ (Komiya [9]), and $\Omega_{*}(X, A, \tau)$ for maps of odd prime period $(X, A, \tau)(\mathrm{Wu}[17])$.

The main object of the present paper is to apply Komiya's theories to the geometrical determination of the oriented bordism algebras $\hat{\Omega}_{*}^{+}\left(Z_{2}\right)$ of all orientationpreserving free differentiable involutions and $\hat{\Omega}_{*}^{-}\left(Z_{2}\right)$ of all orientation-reversing free differentiable involutions. (Compare with the semi-geometric methods in Stong [11, Chapter VIII]).

We also remark in this pape $r$ that the equivariant oriented and weakly complex theories of Wu, together with Mišcenko's theorem [10, Appendix 1], give rise to a simple proof of the structure theorem for $\Omega_{*}\left(Z_{p}\right)[2], U_{*}\left(Z_{m}\right), U^{*}\left(Z_{m}\right)$ ([3], [5], [6], [7]) and $K^{0}\left(L^{n}(m)\right)[8]$. These results are applied to the nonexistence problem for equivariant maps.

In $\S 1$, we define the bordism groups $\hat{\Omega}_{*}^{+}(X, \tau)$ and $\hat{\Omega}_{*}^{-}(X, \tau)$, and then introduce the external product and the Pontrjagin product in these theories.

In $\$ \$ 2$ and 3, we give two kinds of direct sum decompositions of $\hat{\Omega}_{*}^{+}\left(S^{n}, a\right)$ and $\hat{\Omega}_{*}^{+}\left(S^{n}, a\right)$ into isomorphic copies of $\hat{\Omega}_{*}^{-}\left(S^{1}, a\right)$. Deviating slightly from the

Received by the editors July 28, 1971 and, in revised form, February 4, 1972.

AMS (MOS) subject classifications (1970). Primary 57D75, 57D85, 57D90; Secondary 55B20, 55C35, $55 \mathrm{G} 37$.

Key words and phrases. Oriented bordism, weakly complex bordism, free periodic maps, equivariant bordism theory, free differentiable involutions, equivariant maps, Pontrjagin product, Smith homomorphism, cobordism characteristic classes, formal group law, logarithm of a formal group, Miščenko series. 
method in [11], we show explicitly how to construct the elements of $\hat{\Omega}_{*}^{+}\left(S^{n}, a\right)$ and $\hat{\Omega}_{*}^{-}\left(S^{n}, a\right)$ from those of $\hat{\Omega}_{*}^{-}\left(s^{1}, a\right)$. This permits us to deduce the multiplicative structures of $\hat{\Omega}_{*}^{+}\left(Z_{2}\right)$ and $\hat{\Omega}_{*}^{-}\left(Z_{2}\right)$ in $\S 5$.

And in $\$ 4$, the $\Omega_{*}$-algebra structure of $\hat{\Omega}_{*}^{-}\left(S^{1}, a\right)$ is explicitly presented. It was known to be isomorphic as a $Z_{2}$-algebra to Wall's subalgebra $W_{*}$ of $\Re_{*}[16]$.

$\S 5$ is devoted to the study of $\Omega_{*}$-algebra structures of $\hat{\Omega}_{*}^{+}\left(Z_{2}\right)$ and $\hat{\Omega}_{*}^{-}\left(Z_{2}\right)$ with respect to the Pontrjagin product. We present minimal sets of algebra generators for $\hat{\Omega}_{*}^{+}\left(Z_{2}\right)$ and $\hat{\Omega}_{*}^{-}\left(Z_{2}\right)$ together with some explicit multiplication formulas.

In $\$ 6$ we treat the odd period and weakly complex cases in parallel. We combine the technique of $\$ 2$ with Kamata's idea in [7] of applying Miščenko series to $U_{*}\left(L^{n}(m)\right)$. We also remark that the multiplicative structures of $\Omega_{*}\left(Z_{k}\right)(k \geq 3$, odd) and $U_{*}\left(Z_{m}\right)(m \geq 2)$ with respect to the Pontrjagin products are trivial.

Finally, in $\$ 7$, we apply the results on $U_{*}\left(L^{n}(m)\right)$ obtained in the preceding section to a nonexistence problem for some equivariant maps considered in [15].

The author is indebted to Professor Fuichi Uchida, Mr. Katsuhiro Komiya and Professor Ching-Mu Wu for many suggestions concerning these ideas. And the author wishes to express his gratitude to Professors Minoru Nakaoka and Akio Hattori for valuable suggestions and constant encouragements. Thanks are also due to Professor Robert E. Stong and the referee who informed me of many known results in this field which permitted improvements in the presentation, especially of $\$ 2$.

1. Free equivariant oriented bordism groups. A free equivariant orientationpreserving bordism class of $(X, \tau)$, where $X$ is a topological space and $\tau: X \rightarrow X$ is a continuous map such that $\tau^{2}=\mathrm{id}$, is an equivalence class of triples $(M, \mu, f)$ with $M$ a closed oriented differentiable manifold, $\mu: M \rightarrow M$ a fixed-point free differentiable involution which preserves the orientation of $M$, and $f:(M, \mu) \rightarrow$ $(X, \tau)$ a continuous equivariant map $(\tau \circ f=f \circ \mu)$. Two triples $(M, \mu, f)$ and $\left(M^{\prime}, \mu^{\prime}, f\right)$ are equivalent, or bordant if the re is a triple $(W, \nu, g)$ such that $W$ is a compact oriented differentiable manifold with $\partial W=M \cup\left(-M^{\prime}\right), \nu: W \rightarrow W$ is a fixed-point free, orientation-preserving differentiable involution restricting to $\mu$ on $M$ and $\mu^{\prime}$ on $M^{\prime}$, and $g:(W, \nu) \rightarrow(X, \tau)$ is a continuous equivariant map $(\tau \circ g=g \circ \nu)$ restricting to $f$ on $M$ and $f^{\prime}$ on $M^{\prime}$.

The set of free equivariant orientation-preserving bordism classes $(X, \tau)$ becomes a graded $\Omega_{*}$-module in the usual fashion and we denote this module by $\hat{\Omega}_{*}^{+}(X, \tau)([9],[12])$.

A similar graded $\Omega_{*}$-module is obtained by replacing orientation-preserving everywhere in the above definition by orientation-reversing. This module one denotes $\hat{\Omega}_{*}^{-}(X, \tau)$ [9].

Letting $X$ be a point and $\tau$ the identity map, this reduces to the oriented 
analogue of the situation studied by Conner-Floyd [2]. Thus we write $\hat{\Omega}_{*}^{+}\left(Z_{2}\right)$ for $\hat{\Omega}_{*}^{+}(p t, 1)$ and $\hat{\Omega}_{*}^{-}\left(Z_{2}\right)$ for $\hat{\Omega}_{*}^{-}(p t, 1)$, combining the notations of Conner-Floyd [2] and Stong [12].

For every involution $(X, \tau)$, there is the obvious equivariant map $(X, \tau) \rightarrow$ $(p t, 1)$, inducing the homomorphism $\epsilon: \hat{\Omega}_{*}^{+}(X, \tau) \rightarrow \hat{\Omega}_{*}^{+}(p t, 1)$ and $\epsilon: \hat{\Omega}_{*}^{-}(X, \tau) \rightarrow$ $\hat{\Omega}_{*}^{-}(p t, 1)$. We call both of them the equivariant augmentation bomomorphism.

Remark. We will hereafter use the notations like $\epsilon: \hat{\Omega}_{*}^{ \pm}(X, \tau) \rightarrow \hat{\Omega}_{*}^{ \pm}(p t, 1)$ to avoid repeating two formulas which are identical except for the signs.

Let $(X, \tau)$ and $(Y, \sigma)$ be involutions. Then both $\tau \times 1$ and $1 \times \sigma$ induce the same involution on $X \times Y / \tau \times \sigma$.

Lemma 1.1. The pairings

$$
\lambda: \hat{\Omega}_{*}^{ \pm}(X, \tau) \otimes_{\Omega_{*}} \hat{\Omega}_{*}^{ \pm}(Y, \sigma) \rightarrow \hat{\Omega}_{*}^{ \pm}(X \times Y / \tau \times \sigma, 1 \times \sigma)
$$

sending $[M, \mu, f] \otimes[N, \nu, g]$ to $[M \times N / \mu \times \nu, 1 \times \nu, f \times g / \mu \times \nu]$ are well-defined $\Omega_{*}$-module homomorphisms which are natural with respect to equivariant maps $b$ : $(X, \tau) \rightarrow\left(X^{\prime}, \tau^{\prime}\right)$ and $b^{\prime}:(Y, \sigma) \rightarrow\left(Y^{\prime}, \sigma^{\prime}\right)$.

The proof of the lemma is straightforward. So we omit the proof. We call the above pairings the external products.

Let $(X, \tau)$ be an involution. Then $\tau$ induces a $Z_{2} \times Z_{2}$-action on $X \times X$ by $\{\tau \times \tau, \tau \times 1,1 \times \tau, 1 \times 1\}$ and also on $X$ via the addition homomorphism $Z_{2} \times Z_{2}$ $\rightarrow Z_{2}$ and by $r$.

Definition 1.2. In case there is a continuous map $\phi: X \times X \rightarrow X$ which is equivariant with respect to the above-mentioned $Z_{2} \times Z_{2}$-actions, we call $\phi a$ multiplication map with respect to $\tau$. Notice that $\phi$ induces a $Z_{2}$-equivariant map $\hat{\phi}:(X \times X / \tau \times \tau, 1 \times \tau) \rightarrow(X, \tau)$. Associative and commutative multiplication maps and multiplication maps with unit are to be considered in the usual sense and we omit their detailed definition.

Examples 1.3. (1) The constant map $\phi_{P}: p t \times p t \rightarrow p t$ is an associative and commutative multiplication map with unit with respect to the trivial involution.

(2) Let $S^{1}=\{Z \in C ;|Z|=1\}$ be the unit sphere in $C$ and $a: S^{1} \rightarrow S^{1}$ be the antipodal involution: $a(Z)=-Z$. Then the map $\mu: S^{1} \times S^{1} \rightarrow S^{1}$ defined by $\mu\left(Z, Z^{\prime}\right)^{\prime}=Z \cdot Z^{\prime}$ is an associative and commutative multiplication map with unit with respect to $a$.

Definition 1.4. Let $\phi: X \times X \rightarrow X$ be a multiplication map with respect to an involution $(X, \tau)$. The composites of the external products of 2.1 by the induced $\Omega_{*}$-module homomorphisms $\hat{\phi}_{*}$

$$
\hat{\Omega}_{*}^{ \pm}(X, \tau) \otimes_{\Omega_{*}} \hat{\Omega}_{*}^{ \pm}(X, \tau) \rightarrow \hat{\Omega}_{*}^{ \pm}(X \times X / \tau \times \tau, 1 \times \tau) \rightarrow \hat{\Omega}_{*}^{ \pm}(X, \tau)
$$


give rise to product operations in $\hat{\Omega}_{*}^{+}(X, \tau)$ and $\hat{\Omega}_{*}^{-}(X, \tau)$. We call them the Pontrjagin product with respect to the multiplication map $\phi$. In this case, $\hat{\Omega}_{*}^{+}(X, \tau)$ and $\hat{\Omega}_{*}^{-}(X, \tau)$ become graded algebras over $\Omega_{*}$.

2. First direct sum decompositions. Let $(N, \tau)$ be a free involution. Suppose $(L, \tau \mid L)$ is an invariant subspace of $(N, \tau)$ with a given finite dimensional $C^{\infty}$. manifold structure on $L$ making $\tau \mid L$ differentiable and with an invariant neighbourhood which is equivariantly homeomorphic to $(L \times[-1,1], \tau \mid L \times(-1))$ and $L \times\{0\}=L$.

Proposition 2.1. There is an exact triangle of $\Omega_{*}$-module homomorphisms

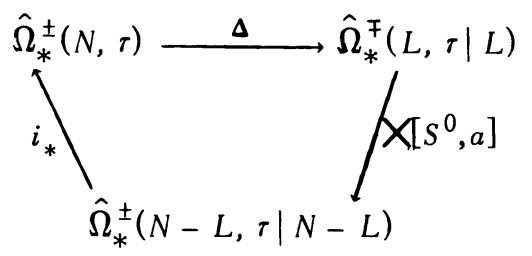

where $\Delta$ denotes the map sending $[M, \mu, f]$ with $f$ t-regular on $L$ to $\left[f^{-1}(L)\right.$, $\left.\mu\left|f^{-1}(L), f\right| f^{-1}(L)\right], i_{*}$ the homomorphism induced by the inclusion and $X\left[S^{0}, a\right]$ the map sending $[M, \mu, f]$ to $[M \times \partial[-1,1], \mu \times(-1), \iota \circ(f \times 1)]$ with $\iota: L \times \partial[-1,1]$ $\subset L \times[-1,1] \subset N$.

We call $\Delta$ the Smith bomomorphism. This proposition is a variant of the Smith exact sequence in [9], [12] and the proof is analogous. It was pointed out by the referee that the exact sequence of the pair $(N, N-L)$ together with a Thom isomorphism $\hat{\Omega}_{*}^{ \pm}(N, N-L, \tau) \cong \hat{\Omega}_{*}^{\mp}(L, \tau \mid L)$ given by $t$-regularity supplies an alternative proof.

Let $(X, \tau)$ be an involution. Denote by $E^{n} X$ the $n$-fold unreduced suspension of $X$, i.e.

$$
E^{n} X=D^{n} \times X /(s, x) \sim\left(s, x^{\prime}\right): \quad s \in \partial D^{n}, x, x^{\prime} \in X
$$

and define $E^{n}(\tau): E^{n} X \rightarrow E^{n} X$ by $E^{n}(\tau)[d, x]=[a(d), \tau(x)]$, where $a$ is the antipodal map. Let $(M, \mu, f)$ be a representation of a class in $\hat{\Omega}_{k}^{-}(X, \tau)$. Let us define $D^{n}(M), D^{n}(\mu)$ and $D^{n}(f)$ as follows:

$$
D^{n}(M)=D^{n} \times M /(s, x) \sim(s, \mu(x)): \quad s \in \partial D^{n}, x \in M,
$$

$D^{n}(\mu): D^{n}(M) \rightarrow D^{n}(M)$ is defined by $D^{n}(\mu)[d, x]=[a(d), \mu(x)]$ and $D^{n}(f): D^{n}(M)$ $\rightarrow E^{n} X$ by $D^{n}(f)[d, x]=[d, f(x)]$.

Proposition 2.2. The map $D^{n}: \hat{\Omega}_{*}^{-}(X, \tau) \rightarrow \hat{\Omega}_{*}^{(-1)^{n+1}}\left(E^{n} X, E^{n}(\tau)\right)$ sending

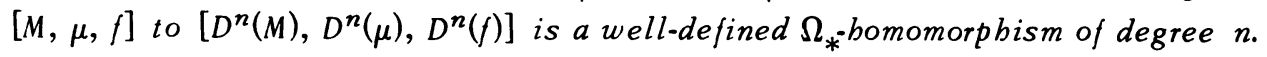


In case $(X, \tau)$ is a free $C^{\infty}$-involution on a finite dimensional $C^{\infty}$-manifold and $n=1$ or in case $(X, \tau)=\left(S^{n}, a\right)$ and $n \geq 1$, we have $\Delta \circ D^{n}=D^{n-1}(n \geq 1)$, where $\Delta$ is the Smith homomorphism of 2.1 with respect to $\left(E^{n-1} X, E^{n-1}(\tau)\right) \subset\left(E^{n} X\right.$, $\left.E^{n}(\tau)\right)$.

The proof is straightforward from the definition. We call $D^{n}$ the n-fold sus. pension of the first kind. This notion is due to Uchida.

Corollary 2.3. Let $\tau: L \rightarrow L$ be a differentiable involution on a finite dimensional $C^{\infty}$-manifold. L. (1) There is a split short exact sequence of $\Omega_{*}$-homomorphisms

$$
\cap \rightarrow \Omega_{*} \stackrel{\left[S^{0}, a\right]}{\longrightarrow} \hat{\Omega}_{*}^{+}\left(E^{1} L, E^{1}(\tau)\right) \stackrel{\Delta}{\stackrel{\Delta)^{1}}{\rightleftarrows}} \hat{\Omega}_{*}^{-}(L, \tau) \rightarrow 0 .
$$

(2) There is an exact sequence of $\Omega_{*}$-bomomorphisms

$$
\Omega_{*} \stackrel{X^{2}}{\longrightarrow} \Omega_{*} \stackrel{\left[S^{0}, a\right]}{\longrightarrow} \hat{\Omega}_{*}^{-}\left(E^{1} L, E^{1}(\tau)\right) \stackrel{\Delta}{\longrightarrow} \hat{\Omega}_{*}^{+}(L, \tau) \stackrel{\rho}{\longrightarrow} 2 \Omega_{*} \rightarrow 0 .
$$

Here the $\left[S^{0}, a\right]$ denote the composite maps

$$
\Omega_{*} \underset{\Omega_{*}^{ \pm}}{\longrightarrow}\left(S^{0}, a\right) \stackrel{i_{*}}{\longrightarrow} \hat{\Omega}_{*}^{ \pm}\left(E^{1} L, E^{1}(\tau)\right)
$$

with $i(+1)=[+1, x]$ and $i(-1)=[-1, x]$ and $\rho$ is the map sending $[M, \mu, f]$ to $[M]$.

Proof. The fact that Image $\rho=2 \Omega_{*}$ was proved in [2]. Since $\left(E^{1} L-L, E^{1}(\tau)\right)$ is equivariantly homotopy equivalent to $\left(S^{0}, a\right)$, the rest follows from 2.1 and 2.2.

Lemma 2.4. (1) Let $(X, \tau)$ be a fixed-point free involution. The map $\eta$ : $\hat{\Omega}_{*}^{+}(X, \tau) \rightarrow \Omega_{*}(X / \tau)$ sending $[M, \mu, f]$ to $[M / \mu, f / \mu]$ is a well-defined isomorphis $m$ of $\Omega_{*}$-modules.

(2) Let $(L, \sigma)$ be a fixed-point free differentiable involution on a finite dimensional $C^{\infty}$-manifold. The map

$$
\eta \circ D^{1}: \hat{\Omega}_{*}^{-}(L, \sigma) \rightarrow \hat{\Omega}_{*}^{+}\left(E^{1}{ }_{i}, E^{1}(\sigma)\right) \underset{\simeq}{\simeq} \Omega_{*}\left(E^{1} L / E^{1}(\sigma)\right)
$$

is a well-defined $\Omega_{*}$-isomorphism onto $\widetilde{\Omega}_{*}\left(E^{1} L / E^{1}(\sigma)\right)$.

Part (1) is well known and (2) is a result of Atiyah in [1]. The proof of (1) is completely analogous to that of Theorem 19.1 in [2]. To see that the image of $\eta \circ D^{1}$ lies in $\widetilde{\Omega}_{*}\left(E^{1} L / E^{1}(\sigma)\right)$, one has only to observe that $D^{1}(M)$ bounds $D_{+}^{2}(M)=$ $\left\{[d, x] \in D^{2}(M) ; d \in D^{2} \cap H_{+}^{2}\right\}$. Then part (2) is a paraphrase of 2.3(1).

Now, as an analogue of Theorem 3 and 4 (3) of $W u[17]$, we obtain the following exact sequence.

Proposition 2.5. (See Stong [11, p. 175].) There is a split short exact sequence of $\Omega_{*}$-modules, for each $n \geq 2$ : 


$$
0 \rightarrow \hat{\Omega}_{*}^{-}\left(S^{1}, a\right) \stackrel{i_{*}}{\longrightarrow} \hat{\Omega}_{*}^{-}\left(S^{n}, a\right) \underset{D^{2}}{\stackrel{\Delta^{2}}{\rightleftarrows}} \hat{\Omega}_{*}^{-}\left(S^{n-2}, a\right) \rightarrow 0 .
$$

Proof. The equality $\Delta^{2} \circ D^{2}=$ id follows from 2.2. The exactness at $\hat{\Omega}_{*}^{-}\left(s^{n}, a\right)$ is easily proved geometrically as in [17]. The fact that $i_{*}$ is monomorphic can be seen by utilizing the preceding lemma together with the collapsibility of the bordism spectral sequence for $R P(n)$ [2, Theorem 15.2].

From this proposition and 2.3(1), we obtain the direct sum decompositions of $\hat{\Omega}_{*}^{ \pm}\left(s^{n}, a\right)$ such as

$$
\hat{\Omega}_{*}^{-}\left(S^{2 n+1}, a\right)=\bigoplus_{n \geq i \geq 0} D^{2 i} \hat{\Omega}_{*}^{-}\left(S^{1}, a\right) .
$$

But we prefer another kind of decompositions which we will present in the next section.

3. Second direct sum decompositions. For the sake of the description of multiplicative structures in $\hat{\Omega}_{*}^{ \pm}\left(Z_{2}\right)$, we give another kind of suspension operators in this section.

Regard $S^{2 n+1}$ as the unit sphere in the complex $(n+1)$-space $C^{n+1}$, and let $S_{0}^{2 n}, S^{2 n}$ and $S^{2 n-1}$ denote the submanifold of $S^{2 n+1}$ defined by

$$
\begin{aligned}
s_{0}^{2 n} & =\left\{\left(Z_{0}, Z_{1}, \cdots, Z_{n}\right) \in S^{2 n+1} \mid Z_{0} \text { is real }\right\}, \\
S^{2 n} & =\left\{\left(Z_{0}, Z_{1}, \cdots, Z_{n}\right) \in S^{2 n+1} \mid Z_{n} \text { is real }\right\} ; \\
S^{2 n-1} & =\left\{\left(Z_{0}, Z_{1}, \cdots, Z_{n}\right) \in S^{2 n+1} \mid Z_{n}=0\right\} .
\end{aligned}
$$

Let $\mu: S^{2 n+1} \times S^{1} \rightarrow S^{2 n+1}$ be the map defined by $\mu\left(\left(Z_{0}, Z_{1}, \cdots, Z_{n}\right), Z\right)=$ $\left(Z_{0} Z, Z_{1} Z, \cdots, Z_{n} Z\right)$. Then, $\mu$ induces an equivariant map $\hat{\mu}:\left(S^{2 n+1} \times S^{1} / a \times a\right.$, $a \times 1) \rightarrow\left(S^{2 n+1}, a\right)$ which is $t$-regular on $S^{2 n-\epsilon}(\epsilon=0,1)$, and $\hat{\mu}^{-1}\left(S^{2 n-1}\right)$ $=\left(S^{2 n-1} \times S^{1 / a} \times a\right)$ (Uchida [14]).

Definition and notation 3.1. (1) For an element $x$ in $\hat{\Omega}_{*}^{ \pm}\left(S^{n}, a\right)$, we also denote by $x$ the element in $\hat{\Omega}_{*}^{ \pm}\left(S^{n+k}, a\right)$ for $k \geq 1$ which should be denoted by $i_{*}(x)$. And denote by $\left[S^{n}, a\right]$ the element which should be denoted by $\left[S^{n}, a\right.$, id].

(2) For each $n \geq 0$, define an $\Omega_{*}$-module homomorphism

$$
E^{2 n}: \hat{\imath}_{*}^{-}\left(S^{1}, a\right) \rightarrow \hat{\Omega}_{*}^{-}\left(S^{2 n+1}, a\right)
$$

of degree $2 n$ by letting $E^{2 n}(x)$ be the image of $\left[S^{2 n}, a\right] \otimes x$ mapped by the composite $\left\{\hat{\mu} \mid\left(S_{0}^{2 n} \times S^{1} / a \times a\right)\right\}_{*} \circ(\theta \times \mathrm{id} / a \times a)_{*} \circ \lambda$ :

$$
\begin{aligned}
\hat{\mathbf{\Omega}}_{*}^{-}\left(S^{2 n}, a\right) \otimes_{\boldsymbol{\Omega}_{*}} & \hat{\mathbf{\Omega}}_{*}^{-}\left(S^{1}, a\right) \rightarrow \hat{\mathbf{2}}_{*}^{-}\left(S^{2 n} \times S^{1} / a \times a, a \times 1\right) \\
& \rightarrow \hat{\mathbf{\Omega}}_{*}^{-}\left(S_{0}^{2 n} \times S^{1} / a \times a, a \times 1\right) \rightarrow \hat{\mathbf{\Omega}}_{*}^{-}\left(S^{2 n+1}, a\right)
\end{aligned}
$$


where $\lambda$ is the external product and $\theta:\left(s^{2 n}, a\right) \rightarrow\left(s_{0}^{2 n}, a \mid s_{0}^{2 n}\right)$ is the equivariant diffeomorphism defined by $\theta\left(Z_{0}, Z_{1}, \cdots, Z_{n-1}, Z_{n}\right)=\left(Z_{n}, Z_{1}, \cdots, Z_{n-1}, Z_{0}\right)$. Obviously, $E^{0}=$ id and $E^{2 n}\left[s^{0}, a\right]=\left[s^{2 n}, a\right]$.

(3) For each $n \geq 0$, define an $\Omega_{* \text {-module homomorphism }}$

$$
E^{2 n+1}: \hat{\Omega}_{*}^{-}\left(S^{1}, a\right) \rightarrow \hat{\Omega}_{*}^{+}\left(S^{2 n+2}, a\right)
$$

of degree $2 n+1$ by $E^{2 n+1}=. \circ E^{2 n+2}$. Obviously, $E^{2 n+1}\left[S^{0}, a\right]=\left[S^{2 n+1}, a\right]$.

Lemma 3.2. $\Delta \circ E^{n}=E^{n-1}(n \geq 1)$.

Proof. It suffices to prove $\Delta^{2} \circ E^{2 n}=E^{2 n-2}$ for $n \geq 1$. This follows from the fact that $\hat{\mu}^{-1}\left(S^{2 n-1}\right)=\left(S^{2 n-1} \times S^{1} / a \times a\right)$ and the definition of $E^{2 n}$ and $E^{2 n-2}$.

Corollary 3.3.

$$
\begin{aligned}
\hat{\Omega}_{*}^{-}\left(S^{2 n+1}, a\right) & =\bigoplus_{n \geq i \geq 0} E^{2 i} \hat{\Omega}_{*}^{-}\left(S^{1}, a\right) . \\
\hat{\Omega}_{*}^{-}\left(S^{2 n}, a\right) & =\left\{\bigoplus_{n-1 \geq i \geq 0} E^{2 i} \hat{\Omega}_{*}^{-}\left(S^{1}, a\right)\right\} \oplus \Omega_{*}\left\{\left\{\left[S^{2 n}, a\right]\right\}\right\} . \\
\hat{\Omega}_{*}^{-}\left(Z_{2}\right) & \cong \hat{\Omega}_{*}^{-}\left(S^{\infty}, a\right)=\bigoplus_{i \geq 0} E^{2 i} \hat{\Omega}_{*}^{-}\left(S^{1}, a\right) .
\end{aligned}
$$

$\hat{\Omega}_{*}^{+}\left(S^{2 n+1}, a\right)$

$$
=\Omega_{*}\left\{\left\{\left[Z_{2}, Z_{2}\right]\right\}\right\} \oplus\left\{\bigoplus_{n-1 \geq i \geq 0} E^{2 i+1} \hat{\Omega}_{*}^{-}\left(s^{1}, a\right)\right\} \oplus \Omega_{*}\left\{\left\{\left[s^{2 n+1}, a\right]\right\}\right\} .
$$

$$
\begin{aligned}
& \hat{\Omega}_{*}^{+}\left(S^{2 n}, a\right)=\Omega_{*}\left\{\left\{\left[Z_{2}, Z_{2}\right]\right\}\right\} \oplus\left\{\underset{n-1 \geq i \geq 0}{\bigoplus} E^{2 i+1} \hat{\Omega}_{*}^{-}\left(S^{1}, a\right)\right\} . \\
& \hat{\Omega}_{*}^{+}\left(Z_{2}\right) \cong \hat{\Omega}_{*}^{+}\left(S^{\infty}, a\right)=\Omega_{*}\left\{\left\{\left[Z_{2}, Z_{2}\right]\right\}\right\} \oplus\left\{\underset{i \geq 0}{\bigoplus} E^{2 i+1} \hat{\Omega}_{*}^{-}\left(s^{1}, a\right)\right\} .
\end{aligned}
$$

Here $\Omega_{*}\left\{\left\{\left[{ }^{*}\right]\right\}\right\}$ denotes the $\Omega_{*^{-}}$free module generated by a class [*] and $\left[Z_{2}, Z_{2}\right]$ is the class of the action of $Z_{2}$ on itself by the addition homomorphism.

Proof. From 2.5 and 3.2, (1) and (2) are easily obtained by induction on $n$. Then (4) and (5) follow from 2.3(1) and 3.2. Now $\hat{\Omega}_{*}^{ \pm}\left(Z_{2}\right) \cong \hat{\Omega}_{*}^{ \pm}\left(s^{\infty}, a\right)$ is a paraphrase of $[2,(19.1)]$. Therefore

$$
\hat{\Omega}_{*}^{ \pm}\left(Z_{2}\right) \cong \hat{\Omega}_{*}^{ \pm}\left(\bigcup_{i \geq 0}\left(S^{i}, a\right)\right)=\frac{\text { Dir. } \operatorname{Lim}}{i} \hat{\Omega}_{*}^{ \pm}\left(S^{i}, a\right) .
$$

Recalling that $\left[S^{i}, a\right]=E^{i}\left[S^{0}, a\right]$, (3) and (6) follow from (1), (2) and (4), (5). 
4. Determination of $\hat{\Omega}_{*}^{-}\left(S^{1}, a\right)$. In this section, we determine the $\Omega_{*}$-algebra structure of $\hat{\Omega}_{*}^{-}\left(S^{1}, a\right)$. In view of Corollary 3.3 , this determines completely the $\Omega_{*}$-module structures of $\hat{\Omega}_{*}^{ \pm}\left(S^{n}, a\right)$.

Let $\mathbb{C}_{*}=Z_{2}\left[X_{2 k-1}, X_{2 k} ; k \neq 2^{i},\left(X_{2}\right)^{2}\right]$ be the polynomial subalgebra of $\Re_{*}$ defined by Wall in [16]. Regard $\hat{\Omega}_{*}^{-}\left(s^{1}, a\right)$ as a ring via the Pontrjagin product with respect to the multiplication map $\mu$ of 1.3(2).

Lemma 4.1 (Wall [16], Stong [11]). The map $\eta: \hat{\Omega}_{*}^{-}\left(S^{1}, a\right) \rightarrow \Re_{*}$, sending $[M, \mu, f]$ to $[M / \mu]$, is a ring isomorpbism onto $\mathbb{i}_{*}$.

Proof. It is clear by definition that $\eta$ is a well-defined ring homomorphism. From the definition of $i_{*}$ in [16], it is also clear that $\eta$ is an epimorphism onto $0_{*}$. Considering the orientation covering of an unoriented manifold, one sees that $\eta_{\infty}: \hat{\Omega}_{*}^{-}\left(S^{\infty}, a\right) \rightarrow \Re_{*}$ defined by $\eta_{\infty}[M, \mu, f]=[M / \mu]$ is a bijection. Therefore $\eta=\eta_{\infty} \circ i_{*}$ is monic since $i_{*}$ is so by $3.3(3)$.

Now we proceed to the description of $\hat{\Omega}_{*}^{-}\left(S^{1}, a\right)$ as an $\Omega_{*}$-algebra.

Notation 4.2 (cf. [16]). (1) Let $\pi$ denote the set of all partitions $\omega=\left(a_{1}\right.$, $\left.a_{2}, \cdots, a_{r}\right)$ with unequal parts $a_{i}$ none of which is a power of 2 . And let $|\omega|=r$ be the length of $\omega$.

(2) For partitions $\omega, \omega^{\prime} \in \pi$, let $\omega \cap \omega^{\prime} \in \pi$ be their intersection i.e. the partition whose parts belong to both $\omega$ and $\omega^{\prime}$. Also $\omega \ominus \omega^{\prime} \in \pi$ be their symmetric difference, i.e. the partition whose parts belong to either $\omega$ or $\omega^{\prime}$ but not to both.

(3) For a partition $\omega=\left(a_{1}, a_{2}, \cdots, a_{r}\right)$, let $\omega_{i}$ denote the partition obtained from $\omega$ by omitting $a_{i}$

With these notations, Wall's result on $\Omega_{*}$ can be stated as follows.

Theorem 4.3 (Wall [16]). (1) The ring structure of $\Omega_{*}$ can be described by the following polynomial presentation:

$$
\begin{aligned}
& 0 \rightarrow g\left[2 g_{\omega} \sum_{i} g_{a_{i}} g_{\omega_{i}} ;|\omega| \geq 3,\left(\sum_{i} b_{\omega_{i} \cap \omega^{\prime}} g_{a_{i}} g_{\omega_{i}} \Theta \omega^{\prime}\right)-g_{\omega} g_{\omega^{\prime}}\right] \\
& \stackrel{\kappa}{\longrightarrow} Z\left[b_{4 k} ; k \geq 0, g_{\omega} ; \omega \in \pi\right] \stackrel{\lambda}{\rightarrow} \Omega_{*} \rightarrow 0,
\end{aligned}
$$

where $Z[\cdots]$ denotes the polynomial ring over $Z$ with generating set $\{\cdots\}$, $g[\ldots]$ the ideal generated by $\{\ldots\}, \kappa$ the natural inclusion and $\lambda$ is the quotient bomomorphism. (For representing manifolds of $g_{\omega}$, see [16].)

(2) The generators of $\Omega_{*}$ given above are irredindant and the relations are independent.

Definition 4.4. For each partition $\omega \in \pi$, define an element $W_{\omega}$ in $\hat{\Omega}_{2\|\omega\|}\left(S^{1}, a\right)$ by $W_{\omega}=\eta^{-1}\left(X_{\omega}\right)$, where $\eta$ is the isomorphism of 4.1, 
$X_{\omega}=X_{2 a_{1}} \cdots X_{2 a_{r}}$ for $\omega=\left(a_{1}, a_{2}, \cdots, a_{r}\right)$ is as defined in [16] and $\|\omega\|=$ $a_{1}+a_{2}+\cdots+a_{x} \cdot$ For convenience, let $W_{\Phi}$ imply $\left[S^{0}, a\right]$ and $b_{\Phi}=1$.

Theorem 4.5. (1) The structure of $\hat{\Omega}_{*}^{-}\left(S^{1}\right.$, a) as an $\Omega_{*}$-module is described by the following $\Omega_{*}$ free presentation:

$$
\begin{aligned}
& 0 \rightarrow \Omega_{*}\left\{2\left[S^{0}, a\right], 2 W_{\omega}, A_{\omega} ;|\omega| \geq 2,{ }^{3} \omega, \omega^{\prime}\right\} \\
& \stackrel{i}{\rightarrow} \Omega_{*}\left\{\left\{\left[S^{0}, a\right], W_{\omega} ; \omega \in \pi\right\}\right\} \stackrel{j}{\rightarrow} \hat{\Omega}_{*}^{-}\left(S^{1}, a\right) \rightarrow 0,
\end{aligned}
$$

where $\Omega_{*}\{\{\ldots\}\}$ denotes the free $\Omega_{*}$ module with generating set $\{\ldots\}, \Omega_{*}\{\ldots\}$ the $\Omega_{*}$-submodule generated by $\{\ldots\}, i$ the natural inclusion, $j$ the quotient bomomorphism and the symbols $A_{\omega}, B_{\omega_{1} \omega^{\prime}}$ stand for the following.

and

$$
A_{\omega}=\sum_{i} g_{a_{i}} W_{\omega_{i}}-g_{\omega}\left[S^{0}, a\right]
$$

$$
{ }^{B} \omega_{\omega} \omega^{\prime}=\sum_{i} b_{\omega_{i} \cap \omega^{\prime}} g_{a_{i}} W_{\omega_{i} \ominus \omega^{\prime}}-g_{\omega} W_{\omega^{\prime}}
$$

(2) The $\Omega_{*}$-module generators given above are irredundant and the relations are independent.

(3) The Pontrjagin products in $\hat{\Omega}_{*}^{-}\left(S^{1}\right.$, a) are described as follows:

(a) $\left[S^{0}, a\right]$ serves as the identity.

(b) $W_{\omega^{\prime}} W_{\omega^{\prime}}=b_{\omega \cap \omega^{\prime}} W_{\omega \Theta \omega^{\prime}}$, in particular, $W_{\omega}=W_{\left(a_{1}\right)} W_{\left(a_{2}\right)} \cdots W_{\left(a_{r}\right)}$ for each $\omega=\left(a_{1}, a_{2}, \cdots, a_{r}\right) \in \pi$.

Proof. (1) It suffices to prove the exactness of the sequence in the theorem, and that is easily derived from 4.1 except for the assertion that ker $j \subset$ Image $i$. So we only prove that part, which requires a cumbersome argument. The verification of the remaining parts are left to the reader.

Denote by $\Lambda_{*}$ the polynomial ring $Z\left[b_{4 k} ; k \geq 0, g_{\omega} ; \omega \in \pi\right]$ given in 4.3 and let $\Lambda_{*}\{\{\ldots\}\}$ denote the free $\Lambda_{*}$-module with generating set $\{\ldots\}$. The ring epimorphism $\lambda: \Lambda_{*} \rightarrow \Omega_{*}$ of 4.3 induces a $\Lambda_{*}$-module epimorphism $\lambda_{*}: \Lambda_{*}\{\{\cdots\}\}$ $\rightarrow \Omega_{*}\left\{\{\ldots\}\right.$, where $\Omega_{*}$ is regarded as a $\Lambda_{*}$-module via $\lambda$. Define a $\Lambda_{*}$-homomorphism $\tilde{\Lambda}: \Lambda_{*}\left\{\left\{\left[s^{0}, a\right], W_{\omega} ; \omega \in \pi\right\}\right\} \rightarrow \Lambda_{*}$ by $\widetilde{\Delta}\left[s^{0}, a\right]=0$ and $\widetilde{\Delta}\left(w_{\omega}\right)=g_{\omega}$. Similarly, let $\Delta^{\prime}: \Omega_{*}\left\{\left\{\left[s^{0}, a\right], W_{\omega} ; \omega \in \pi\right\}\right\} \rightarrow \Omega_{*}$ be the $\Omega_{*}$-homomorphism defined by $\Delta^{\prime}\left[S^{0}, a\right]=0$ and $\Delta^{\prime}\left(W_{\omega}\right)=g_{\omega}$. By definition, $\lambda \circ \tilde{\Delta}=\Delta^{\prime} \circ \lambda_{*}$ and $\Delta \circ j=\left[Z_{2}\right] \circ$ $\Delta^{\prime}$, where $\left[Z_{2}\right]: \Omega_{*} \rightarrow \hat{\Omega}_{*}^{+}\left(S^{0}, a\right)$ is the isomorphism sending $[M]$ to $[M]\left[Z_{2}, Z_{2}\right]$ and $\left[Z_{2}, Z_{2}\right]$ is as defined in 3.3 .

Now suppose $j(x)=0$ for some homogeneous element $x$. Take an element $\tilde{x}$ in $\Lambda_{*}\left\{\left\{\left[S^{0}, a\right], w_{\omega} ; \omega \in \pi\right\}\right\}$ such that $\lambda_{*}(\tilde{x})=x$. Then, $\lambda \circ \tilde{\Delta}(\tilde{x})=\Delta^{\prime} \circ \lambda_{*}(\tilde{x})=$ $\Delta^{\prime}(x)=0$ because $\left[Z_{2}\right] \circ \Delta^{\prime}(x)=\Delta \circ j(x)=0$. Therefore $\tilde{\Delta}(\tilde{x})$ is in Image $\kappa$ by 4.4 , i.e. 


$$
\begin{aligned}
\tilde{\Delta}(\tilde{x})= & \sum_{\omega} \alpha_{\omega} \cdot 2 g_{\omega}+\sum_{|\omega| \geq 3} \beta_{\omega}\left(\sum_{i} g_{a_{i}} g_{\omega_{i}}\right) \\
& +\sum_{\omega, \omega^{\prime}} \gamma_{\omega, \omega^{\prime}}\left\{\sum_{i}\left(b_{\omega_{i} \cap \omega^{\prime}} g_{a_{i}} g_{\omega_{i} \ominus \omega^{\prime}}\right)-g_{\omega} g_{\omega^{\prime}}\right\}
\end{aligned}
$$

for some $\alpha_{\omega}, \beta_{\omega}$ and $\gamma_{\omega, \omega^{\prime}} \in \Lambda_{*}$.

Define an element $\tilde{x}^{\prime}$ in $\Lambda_{*}\left\{\left\{\left[S^{0}, a\right], W_{\omega} ; \omega \in \pi\right\}\right\}$ by

$$
\begin{aligned}
\tilde{x}^{\prime}= & \sum_{\omega} a_{\omega} \cdot 2 W_{\omega}+\sum_{|\omega| \geq 3} \beta_{\omega}\left(\sum_{i} g_{a_{i}} W_{\omega_{i}}-g_{\omega}\left[s^{0}, a\right]\right) \\
& +\sum_{\omega, \omega^{\prime}} \gamma_{\omega, \omega^{\prime}}\left\{\sum _ { i } \left(b_{\omega_{i} \cap \omega^{\prime}} g_{\left.\left.a_{i}{ }_{i}{ }_{\omega_{i}} \Theta \omega^{\prime}\right)-g_{\omega} W_{\omega^{\prime}}\right\}}\right.\right.
\end{aligned}
$$

Then

$$
\tilde{\Delta}\left(\tilde{x}^{\prime}\right)=\tilde{\Delta}(\tilde{x}) \text { and } \lambda_{*}\left(\tilde{x}^{\prime}\right) \in \text { Image } i \text {. }
$$

Now $\tilde{x}-\tilde{x}^{\prime}$ in $\Lambda_{*}\left\{\left\{\left[S^{0}, a\right], W_{\omega} ; \omega \in \pi\right\}\right\}$ can be uniquely expressed as $\tilde{x}-\tilde{x}^{\prime}=\alpha\left[S^{0}, a\right]+\Sigma_{\omega}\left(2 L_{\omega}^{(0)}+L_{\omega}^{(1)}\right) W W_{\omega}$, where $\alpha, L_{\omega}^{(0)}$ and $L_{\omega}^{(1)}$ are polynomials in $\Lambda_{*}$ such that each monomial in $L_{\omega}^{(1)}$ has coefficient one or zero. Then,

and so

$$
\sum_{\omega}\left(2 L_{\omega}^{(0)}+L_{\omega}^{(1)}\right) g_{\omega}=\tilde{\Delta}\left(\tilde{x}-\tilde{x}^{\prime}\right)=0
$$

$$
\sum_{\omega} L_{\omega}^{(1)} g_{\omega}=2 \sum_{\omega}\left(-L_{\omega}^{(0)}\right) g_{\omega}
$$

Since $g_{\omega}$ is a polynomial generator of $\Lambda_{*}$ for each $\omega \in \pi$, the above equality implies that, if $L_{\omega}^{(1)} g_{\omega}$ contains a monomial $\tilde{a} \cdot g_{\omega}\left(\tilde{a} \in \Lambda_{*}\right)$ with coefficient one, there should exist an $\omega^{\prime}$ such that $\omega^{\prime} \neq \omega$ and that $L_{\omega^{\prime}}^{(1)} g_{\omega^{\prime}}$ contains the same monomial with coefficient one. This implies that $\tilde{a}=\tilde{b} \cdot g_{\omega}^{\prime}$ for some monomial $\tilde{b}$ in $\Lambda_{*}$ and that $L_{\omega}^{(1)}$, contains the monomial $\tilde{b} \cdot g_{\omega}$ with coefficient one. By this correspondence $\tilde{b} \cdot g_{\omega} \leftrightarrow \tilde{b} \cdot g_{\omega}$ (not necessarily unique), we can pair all the terms in $\Sigma_{\omega} L_{\omega}^{(1)} W_{\omega}$ as $\Sigma_{\omega} L_{\omega}^{(1)} W_{\omega}=\Sigma_{\omega \neq \omega^{\prime}} K_{\omega, \omega^{\prime}}\left(g_{\omega} W_{\omega^{\prime}}+g_{\omega^{\prime}} W_{\omega}\right)$ for some $K_{\omega, \omega} \in \Lambda_{*} \cdot$

Define an element $\tilde{x}^{\prime \prime}$ in $\Lambda_{*}\left\{\left\{\left[S^{0}, a\right], W_{\omega} ; \omega \in \pi\right\}\right\}$ by $\tilde{x} "=$ $\Sigma_{\omega \neq \omega^{\prime}} K_{\omega, \omega^{\prime}}\left(g_{\omega^{W} \omega^{\prime}}+g_{\omega^{\prime}} W_{\omega}-h_{\omega \cap \omega^{\prime}} g_{\omega \vartheta \omega^{\prime}}\left[s^{0}, a\right]\right)$. Then,

$$
\begin{aligned}
\lambda_{*}\left(\tilde{\tilde{x}}^{\prime \prime}\right) & =\sum_{\omega \neq \omega^{\prime}} \lambda_{*}\left(K_{\omega, \omega^{\prime}}\right)\left(b_{\omega \cap \omega^{\prime}} A_{\omega \vartheta \omega^{\prime}}-B_{\omega, \omega^{\prime}}-B_{\omega^{\prime}, \omega}+\sum_{i} b_{\left(\omega \cap \omega^{\prime}\right)} g_{i} a_{i}\left(2 W_{\omega \vartheta \omega^{\prime}}\right)\right) \\
& \in \text { Image } i .
\end{aligned}
$$


It follows that

$$
\begin{aligned}
x= & \lambda_{*}(\tilde{x})=\lambda_{*}\left(\tilde{x}^{\prime}\right)+\sum_{\omega} \lambda_{*}\left(L_{\omega}^{(0)}\right)\left(2 W_{\omega}\right)+\lambda_{*}\left(\tilde{x}^{\prime \prime}\right) \\
& +\left(\lambda_{*}(\alpha)+\sum_{\omega \neq \omega^{\prime}} \lambda_{*}\left(K_{\omega, \omega^{\prime}}\right) b_{\omega \cap \omega^{\prime}} g_{\omega \ominus \omega^{\prime}}\right)\left[s^{0}, a\right] .
\end{aligned}
$$

By (4.6), (4.7) and (4.8),

$$
\left(\lambda_{*}(a)+\sum_{\omega \neq \omega^{\prime}} \lambda_{*}\left(K_{\omega, \omega^{\prime}}\right) b_{\omega \cap \omega^{\prime}} g_{\omega \ominus \omega^{\prime}}\right)\left[s^{0}, a\right]=j(x)=0
$$

in $\hat{\Omega}_{*}^{-}\left(S^{1}, a\right)$. This implies, by $2.3(2), \lambda_{*}(\alpha)+\Sigma_{\omega \neq \omega^{\prime}} \lambda_{*}\left(K_{\left.\omega_{*} \omega^{\prime}\right) b}\right) b_{\omega} \omega^{\prime} g_{\omega \ominus \omega^{\prime}}=$ $2[M]$ for some $[M]$ in $\Omega_{*^{*}}$. So that last terms in (4.8) can be expressed as [M] $2\left[S^{0}, a\right]$. Therefore $x \in$ Image $i$ and this completes the proof of (1).

(2) is clear from (1).

(3) Part (a) is clear from the definition. Part (b) is proved by 4.1 as follows: $\eta\left(W_{\omega} W_{\omega^{\prime}}\right)=X_{\omega} X_{\omega^{\prime}}=X_{\omega \cap \omega^{\prime}}^{2} \cdot X_{\omega \ominus \omega^{\prime}}=\eta\left(b_{\omega \cap \omega^{\prime}} W_{\omega} \omega_{\omega^{\prime}}\right)$ since $r\left(b_{\omega}\right)=X_{\omega}^{2}$ for $\omega \in \pi[16]$. This completes the proof of the theorem.

5. Multiplicative structures of $\hat{\Omega}_{*}^{+}\left(Z_{2}\right)$ and $\hat{\Omega}_{*}^{-}\left(Z_{2}\right)$. For the calculation of the multiplicative structures of $\hat{\Omega}_{*}^{+}\left(Z_{2}\right)$ and $\hat{\Omega}_{*}^{-}\left(Z_{2}\right)$ with respect to the Pontrjagin products, we need the following two lemmas.

Lemma 5.1. The following sequences are exact for $k \geq 0$ :

$$
\hat{\Omega}_{k}^{+}\left(Z_{2}\right) \stackrel{X^{2}}{\longrightarrow} \hat{\Omega}_{k}^{+}\left(Z_{2}\right) \rightarrow \Re_{k}\left(Z_{2}\right)
$$

and

$$
0 \rightarrow \hat{\Omega}_{k}^{-}\left(Z_{2}\right) \rightarrow \Re_{k}\left(Z_{2}\right)
$$

Proof. The lemma is a direct consequence of the equivariant version of the generalized Rochlin type exact sequence in [9], letting $(X, A, \tau)$ be $(p t, \Phi$, id). Notice that $2 \cdot \hat{\Omega}_{*}^{-}\left(Z_{2}\right)=0$ by 3.3 and $4.5(1)$.

Lemma 5.2. $r\left(W_{\omega}\right)=\left(\partial_{1} X_{\omega}\right)\left[S^{1}, a\right]_{2}+X_{\omega}\left[S^{0}, a\right]_{2}$ in $\Re_{*}\left(Z_{2}\right)$, where $\partial_{1}=$ $r \circ \partial_{3}[16]$ and $\left[S^{i}, a\right]_{2}$ implies $r\left(\left[S^{i}, a\right]\right)$.

Proof. Let $\Delta_{2}$ denote the ordinary Smith homomorphism $\Re_{k}\left(Z_{2}\right) \rightarrow \Re_{k-1}\left(Z_{2}\right)$ [12] and $\epsilon$ be the equivariant augmentation homomorphism. Since $\Delta_{2} \circ r\left(W_{\omega}\right)=$ $r \circ \epsilon \circ \Delta\left(W_{\omega}\right)=r \circ \epsilon\left(g_{\omega} \cdot\left[Z_{2}, Z_{2}\right]\right)=\left(\partial_{1} X_{\omega}\right)\left[S^{0}, a\right]_{2}$,

$$
r\left(W_{\omega}\right)=\left(\partial_{1} X_{\omega}\right)\left[S^{1}, a\right]_{2}+[M]\left[S^{0}, a\right]_{2}
$$


for some $[M] \in \Re_{*}[2]$. But

$$
\begin{aligned}
{[M] } & =c_{*}\left([M] \cdot\left[S^{0}, a\right]_{2}\right)=c_{*} \circ r\left(W_{\omega}\right)-\left(\partial_{1} X_{\omega}\right) c_{*}\left(\left[S^{1}, a\right]_{2}\right) \\
& =\eta\left(W_{\omega}\right)-\left(\partial_{1} X_{\omega}\right) \cdot\left[P_{1}(R)\right]_{2}=X_{\omega}-0=X_{\omega},
\end{aligned}
$$

where $\eta$ is the isomorphism of 4.1. This proves the lemma.

Theorem 5.3. The set $\left\{\left[S^{0}, a\right],\left[S^{2^{i}}, a\right] ; i \geq 1, W_{(k)} ; k \neq 2^{j}\right\}$ supplies a minimal set of generators for the commutative and associative $\Omega_{*}$-algebra with unit $\hat{\Omega}_{*}^{-}\left(Z_{2}\right)$. The multiplicative relations in $\hat{\Omega}_{*}^{-}\left(Z_{2}\right)$ are as follows:

(1) $W_{\omega^{\prime}} W_{\omega^{\prime}}=b_{\omega \cap \omega^{\prime}} W_{\omega \ominus \omega^{\prime}}$, in particular, $W_{\omega}=W_{\left(a_{1}\right)} W_{\left(a_{2}\right)} \cdots W_{\left(a_{r}\right)}$ for $\omega=$ $\left(a_{1}, a_{2}, \ldots, a_{r}\right) \in \pi$. (Here $W_{\Phi}=\left[S^{0}, a\right]$ and $\left.b_{\Phi}=b_{0}.\right)$

(2) $E^{2 n} W_{\omega}=\left[S^{2 n}, a\right] \cdot W_{\omega}$ for eacb $n \geq 0$ and $\omega \in \pi$.

(3) The products of the form $\left[S^{2 n}, a\right] \cdot\left[S^{2 n^{\prime}}, a\right]$ are calculable in theory by 5.1, 5.2 and the results of Uchida [14] on $\Re_{*}\left(Z_{2}\right)$. For example, $\left[s^{2 n}, a\right]^{2}=$ $\left[P_{n}(C)\right]\left[S^{0}, a\right]$ and $\left[S^{2}, a\right] \cdot\left[S^{4}, a\right]=\left[S^{6}, a\right]+W_{(3)}$.

Proof. From 1.3, $\hat{\Omega}_{*}^{-}\left(Z_{2}\right)$ becomes a commutative and associative $\Omega_{*}$-algebra with unit via the Pontrjagin product with respect to $\phi_{P}$. Part (1) follows from 4.5(3), part (2) follows from the definition of $E^{2 n}$, and part (3) is clear. The assertion that the set stated in the theorem generates $\hat{\Omega}_{*}^{-}\left(Z_{2}\right)$ as an $\Omega_{*}$-algebra is easily proved from parts (1), (2), (3) of the theorem and from $3.3,4.5,5.1,5.2$ together with the fact that $\Delta_{2}^{2 n+2 n^{\prime}}\left(\left[S^{2 n}, a\right]_{2} \cdot\left[S^{2 n^{\prime}}, a\right]_{2}\right)=\underset{n}{\left(\begin{array}{c}n+n \\ n\end{array}\right)}\left[S^{0}, a\right]_{2}[13]$. The irredundancy of the generators $\left[S^{0}, a\right]$ and $\left[S 2^{i}, a\right](i \geq 1)$ is clear from parts (1), (2), (3) and the result on $\Re_{*}\left(Z_{2}\right)$ mentioned above. The irredundancy of $W_{(k)}$ $\left(k \neq 2^{j}\right)$ is derived from that of $X_{2 k}$ in $\left\{X_{2 k} ; k \geq 0, X_{2 k-1} ; k \neq 2^{i}(i \geq 0), k \geq 3\right\}$ as a set of polynomial generators for $\Re_{*}$. We leave the details to the reader.

Theorem 5.4. The set $\left\{\left[Z_{2}, Z_{2}\right],\left[S^{2 i+1}, a\right] ; i \geq 0, E^{2 i+1} W_{\omega} ; \omega \in \pi, i \geq 0\right\}$ supplies a minimal set of generators for the commutative and associative $\Omega_{*}$ alge. bra with unit $\hat{\Omega}_{*}^{+}\left(Z_{2}\right)$. The multiplicative relations are as follows:

(1) $\left[S^{2 i+1}, a\right] \cdot\left[S^{2 j+1}, a\right]=0(i, j \geq 0)$.

(2) The products of the form $\left[S^{2 i+1}, a\right] \cdot E^{2 j+1} W_{\omega}$ and $\left(E^{2 i+1} W_{\omega}\right)\left(E^{2 j+1} W_{\omega}\right)$ can be calculable in theory as in the case for $\hat{\Omega}_{*}^{-}\left(Z_{2}\right)$. In particular, $\left(E^{2 i+1} W_{\omega}\right)^{2}$ $=0$ for $\omega \in \pi, i \geq 0$.

The proof is analogous to that of 5.3. So we omit it.

6. The odd period cases and weakly complex cases. In this section we consider the free equivariant oriented (weakly complex, respectively) bordism group $\Omega_{*}(X, \tau)\left(U_{*}(X, \tau)\right)$ of $\mathbb{W u}[17]$ for a pair $(X, \tau)$, where $X$ is a topological space 
and $\tau: X \rightarrow X$ is a continuous periodic map with odd period $p \geq 3$ (with period $m \geq 2$ ). Its definition is completely analogous to that of $\hat{\Omega}_{*}^{+}($,$) given at the be-$ ginning of $\$ 1$, only to replace involution by map of odd period $p \geq 3$ (map of period $m \geq 2$, and orientation by weakly complex structure).

As in $\$ 3$, regard $S^{2 n+1}$ as the unit sphere in $C^{n+1}$. For each integer $m \geq 2$, let $T_{(m)}: S^{2 n+1} \rightarrow S^{2 n+1}$ be the free periodic map defined by $T_{(m)}\left(Z_{0}, \cdots, Z_{n}\right)=$ $\left(\lambda_{(m)} Z_{0}, \cdots, \lambda_{(m)} Z_{n}\right)$ where $\lambda_{(m)}=\exp (2 \pi i / m)$.

Analogously to 2.5 , we can easily modify Theorem 3 and 4(3) of Wu [17] as follows. The proof is omitted.

Theorem 6.1. (1) For each integer $m \geq 2$, the sequence

$$
\begin{aligned}
& 0 \longrightarrow \widetilde{U}_{k}\left(S^{1}, T(m)\right. \stackrel{X^{m}}{\longrightarrow} \widetilde{U}_{k}\left(S^{1}, T(m)\right. \\
& \stackrel{i_{*}}{\longrightarrow} \tilde{U}_{k}\left(S^{2 n+1}, T_{(m)}\right) \\
& \stackrel{\Delta}{\longrightarrow} \tilde{U}_{k-2}\left(S^{2 n-1}, T_{(m)^{\prime}}\right)
\end{aligned}
$$

is exact for each $n \geq 1$, where $i:\left(S^{1}, T(m)\right) \subset\left(S^{2 n+1}, T(m)\right)$ is the natural inclusion, $\tilde{U}_{*}\left(S^{2 i+1}, T(m)\right)$ is the kernel of the ordinary augmentation bomomorphism $U_{*}\left(S^{2 i+1}, T(m)\right) \rightarrow U_{*}\left(S^{2 i+1} / T(m)\right) \rightarrow U_{*}$, and $\Delta$ is the Smith bomomorphism analogous to that of 2.1 .

(2) In case $m$ is an odd integer $\geq 3$, analogous results bold in the oriented case: one can replace $U_{*}()$ of (1) by $\Omega_{*}($ ).

Now denote the quotient spaces $S^{2 n+1} / T(m)$ by $L^{n}(m)$ and the complex projective spaces by $C P(n)$. Let $\left[L^{n-j}(m), i\right]$ be the weakly complex bordism class in $U_{2(n-j)+1}\left(L^{n}(m)\right)$ represented by the natural inclusion map $i: L^{(n-j)}(m) \subset$ $L^{n}(m)$, and $D: U_{2(n-j)+1}\left(L^{n}(m)\right) \rightarrow U^{2 j}\left(L^{n}(m)\right)$ be the Atiyah-Poincare duality [1]. And denote by $\eta$ the canonical complex line bundle over $C P(n), \pi: L^{n}(m)$ $\rightarrow C P(n)$ the natural projection, and $c_{1}(\xi)$ the first Chern class of a complex vector bundle $\xi$ in $U^{*}$-cobordism theory in the sense of Conner-Floyd [4].

Theorem 6.2 (Kamata [7]). With the notations as above, it bolds that $D\left[L^{n-j}(m), i\right]=\left\{c_{1}\left(\pi^{*} \eta\right)\right\}^{j}$ for $m \geq 2, n \geq 1$ and $j \geq 0$.

As in [7], [10], let $F(X, Y)$ be the formal group law defined in $U^{*}[[X, Y]]$ characterised by the property that $c_{1}\left(\xi \otimes \xi^{\prime}\right)=F\left(c_{1}(\xi), c_{1}\left(\xi^{\prime}\right)\right)$ for complex line bundles $\xi$ and $\xi^{\prime}$ over the same $C W$-complex. For each integer $m \geq 2$, define a formal power series $[m]_{F}(X) \in U^{*}[[X]]$ by $[2]_{F}(X)=F(X, X)$ and $[m+1]_{F}(X)=$ $F\left(X,[m]_{F}(X)\right)$. Then $[m]_{F}(x)$ is uniquely expressed as $[m]_{F}(X)=$ $\Sigma_{0 \leq k} V_{k}^{(m)} X^{k+1}$ with $V_{k}^{(m)} \in U^{-2 k}$. Hereafter we identify $U_{*}$ with $U^{*}$ via $D$ and denote by $V_{i}^{(m)}$ the element $D^{-1}\left(V_{i}^{(m)}\right)$ in $U_{*}$. 
Now set $\alpha_{2 k+1}=\left[S^{2 k+1}, T(m), i\right] \in \tilde{U}_{2 k+1}\left(S^{2 n+1}, T(m)\right)(n \geq k \geq 0)$ and $\beta_{2 k+1}=\Sigma_{0 \leq j \leq k} V_{k-j}^{(m)} a_{2 j+1}$. Let $R: U_{*} \rightarrow \Omega_{*}$ and $R: U_{*}\left(S^{2 n+1}, T(m)\right) \rightarrow \Omega_{*}\left(S^{2 n+1}, T(m)\right)$ (in case $m$ is odd) be the homomorphism forgetting a weakly complex structure.

Theorem 6.3 (cf. Conner-Floyd [2], [3], tom Dieck [5], Giffen [6], Kamata [7]). (1) For each integer $m \geq 2, \tilde{U}_{*}\left(S^{2 n+1}, T(m)\right)(n \geq 0)$ is isomorpbic as a $U_{*}$ module to the quotient of the free $U_{*}$ module generated by $\alpha_{1}, \alpha_{3}, \cdots, \alpha_{2 n+1}$ by the sub. module generated by $\beta_{1}, \beta_{3}, \ldots, \beta_{2 n-1}$.

(2) In case $m$ is an odd integer $\geq 3$, analogous results bold in the oriented case: one can replace $\widetilde{U}_{*}(), \alpha_{i}$ and $\beta_{j}$ of (1) by $\widetilde{\Omega}_{*}, R\left(\alpha_{i}\right)$ and $R\left(\beta_{j}\right)$, respectively.

(3) Let $\left[S^{2 n+1} \times Z_{m}, 1 \times T(m), \mu\right]$ be the equivariant bordism class in $U_{2 n+1}\left(S^{2 n+1}, T(m)\right)$ represented by the equivariant map which is the restriction of $\mu: S^{2 n+1} \times S^{1} \rightarrow S^{2 n+1}$ defined in $\$ 3$. Then $\left[S^{2 n+1} \times Z_{m}, 1 \times T(m), \mu\right]=\beta_{2 n+1}$. Equivalently, the bordism class $\left[S^{2 n+1}, \pi_{m}\right] \in \tilde{U}_{*}\left(L^{n}(m)\right)$ represented by the standard m-fold covering map of $S^{2 n+1}$ onto $L^{n}(m)$ is expressed as

$$
\left[S^{2 n+1}, \pi_{m}\right]=\sum_{0 \leq j \leq n} V_{n-j}^{(m)}\left[L^{j}(m), i\right] \quad \text { in } U_{*}\left(L_{n}(m)\right) .
$$

(4) $U^{*}\left(L^{n}(m)\right) \cong \Lambda_{U^{*}}(D[p t, i]) \oplus U^{*}\left[\left[c_{1}\right]\right] /\left(c_{1}^{n+1},[M]_{F}\left(c_{1}\right)\right)$, where $[p t, i] \epsilon$ $U_{0}\left(L^{n}(m)\right)$ is the class of an inclusion map of a point $\Lambda_{U^{*}}()$ is the exterior alge. bra over $U^{*}, c_{1}=c_{1}(\pi * \eta)$, and $(*, *)$ denotes the ideal generated by the set $\{*, *\}$.

Outline of Proof. The proof has nothing new than those of the oriented cases for involutions although there exists no splitting homomorphism as $D^{2}$ here.

(1) It is easily seen that $\tilde{U}_{*}\left(S^{1}, T(m)\right)$ is a free $U_{*}$-module generated by $a_{1}$. Then, the Smith homomorphism argument shows that $a_{2 n+1}$ generates a free $U_{*}$ submodule in $\tilde{U}_{*}\left(S^{2 n+1}, T(m)\right)$ and that $\alpha_{1}, \alpha_{3}, \cdots, \alpha_{2 n+1}$ generates $\tilde{U}_{*}\left(S^{2 n+1}, T(m)\right)$, applying 6.1(1). By the duality in 6.2,

$$
\left.D^{\prime} \cdot 3_{2 k+1}\right)=\sum_{0 \leq j \leq k} V_{k-j}^{(m)} c_{1}^{n-j}=c_{1}^{n-1-k}\left(\sum_{0 \leq j \leq k} V_{j}^{(m)} c_{1}^{j+1}\right)=c_{1}^{n-1-k} \cdot[m]_{F}\left(c_{1}\right)
$$

for $0 \leq k \leq n-1$. But $[m]_{F}\left(c_{1}\right)=c_{1}\left(\{\pi * \eta\}^{m}\right)=c_{1}\left(1_{c}\right)=0$. So $\beta_{2 k+1}=0$ for $0 \leq k \leq n-1$. The assertion that all the module relations are generated by $\beta_{1}$, $\beta_{3}, \cdots, \beta_{2 n-1}$ is easily proved by induction on $n$, applying $6.1(1)$.

(2) From part (1), $R\left(\beta_{2 k+1}\right)=0(0 \leq k \leq n-1)$ in $\tilde{\Omega}_{*}\left(S^{2 n+1}, T(m)\right)$ when $m$ is odd $\geq 3$. The rest of the assertions are proved in the same way as part (1), applying $6.1(2)$.

(3) Let $\tilde{\eta}: U_{*}\left(S^{2 n+1}, T(m)\right) \rightarrow U_{*}\left(L^{n}(m)\right)$ be the natural isomorphism taking orbit spaces. 
In case $n=0, \tilde{\eta}\left(\beta_{1}\right)=\left[S^{1}, \pi_{m}\right]$ is clear by considering the evaluation homomorphism $U_{*}\left(L^{1}(m)\right) \rightarrow H_{*}\left(L^{n}(m) ; Z\right)$.

Assume that part (3) holds for $n-1$. Then $\Delta\left[s^{2 n+1}, \pi_{m}\right]=\left[s^{2 n-1}, \pi_{m}\right]=$ $\tilde{\eta}\left(\beta_{2 n-1}\right)$ in $U_{*}\left(L^{n-1}(m)\right)$. So $\Delta\left(\left[s^{2 n+1}, \pi_{m}\right]-\tilde{\eta}\left(\beta_{2 n+1}\right)\right)=0$ and, by $6.1(1)$, $\left[s^{2 n+1}, \pi_{m}\right]=\tilde{\eta}\left(\beta_{2 n+1}\right)+[M] \tilde{\eta}\left(\alpha_{1}\right)$ for some $[M] \in U_{2 n}$. But $i \circ \pi_{m}: s^{2 n+1} \rightarrow$ $L^{n}(m) \subset L^{n+1}(m)$ bounds $D_{+}^{2 n+2} \subset S^{2 n+3} \rightarrow L^{n+1}(m)$, and so $i_{*}\left[S^{2 n+1}, \pi_{m}\right]=0$ in $U_{*}\left(L^{n+1}(m)\right)$, which implies $[M] \tilde{\eta}\left(\alpha_{1}\right)=0$ in $U_{*}\left(L^{n+1}(m)\right)$ and $[M] \in m U_{*}$. Therefore $[M] \tilde{\eta}\left(\alpha_{1}\right)=0$ holds also in $U_{*}\left(L^{n}(m)\right)$, and this completes the proof of the induction step.

(4) Part (4) directly follows from part (1), applying the duality in 6.2.

Remark 6.4. (1) The $U_{*^{-}}\left(\Omega_{*^{-}}\right)$module structure of $U_{*}\left(Z_{m}\right)$ for $m \geq 2\left(\Omega_{*}\left(Z_{p}\right)\right.$ for odd integer $p \geq 3$ ) follows from the above theorem by taking direct limit. Imposing a complete and Hausdorff topology on $U_{*}\left(Z_{m}\right)[5]$, the cobordism algebra $U^{*}\left(Z_{m}\right)$ is determined from 6.3:4) by taking inverse limit, which deletes the exterior algebra part of $U^{*}\left(L^{n}(m)\right)$.

(2) The Pontrjagin products in $U_{*}\left(Z_{m}\right)$ and $\Omega_{*}\left(Z_{p}\right)(p$ : odd integer $\geq 3)$ are trivial in the sense that $x \cdot y=0$ for any elements in $\widetilde{U}_{*}\left(Z_{m}\right)$ (and $\widetilde{\Omega}_{*}\left(Z_{p}\right)$ ). This follows from the dimensional reason and the fact that $\left[L^{n}(m)\right]=0$ in $U_{*}$.

(3) In case $m=2, U_{*}\left(S^{2 n}, a\right)$ and $U^{*}\left(S^{2 n}, a\right)$ are easily determined by 6.3 , using the cofibration $(R P(2 n), R P(2 n-1))$.

Corollary 6.5 (cf. Kambe [8]). Let $m \geq 2$ be an integer.

(1) $K^{0}\left(L^{n}(m)\right)=Z[[\sigma]] /\left(\sigma^{n+1},(1+\sigma)^{m}-1\right)$, where $\sigma=\pi^{*} \eta-1$.

(2) The Todd genus Todd $\left[V_{i}^{(m)}\right]$ is equal to the binomial coefficient $(-1)^{m+1}\left(\begin{array}{c}m \\ i+1\end{array}\right)$. In particular, $\operatorname{Todd}\left[V_{m-1}^{(m)}\right]=0$ for $i>m-1$.

Proof. Let $c_{i}^{(K)}()$ be the $i$ th Chern class in $K^{*}$-theory defined in [4] and $G(X, Y) \in K^{*}(p t)[[X, Y]]$ be the formal group law characterized by the property $c_{1}^{(k)}\left(\xi \otimes \xi^{\prime}\right)=G\left(c_{1}^{(K)}(\xi), c_{1}^{(K)}\left(\xi^{\prime}\right)\right)$ for line bundles $\xi$ and $\xi^{\prime}$ over the same CW-complex. It is well known that $G(X, Y)=X+Y-X Y$. Then, $[m]_{G}(X)=$ $1-(1-X)^{m}$. Part (1) follows from $6.3(4)$ by considering the natural transformation $\mu_{c}: U^{*}() \rightarrow K^{*}()$ defined in [4]. It is easily seen that $\mu_{c}\left(c_{1}\right)=-\sigma$. Part (2) follows from the fact that $\mu_{c}: U_{*} \cong U_{*}(p t) \rightarrow K^{*}(p t) \cong Z$ is given by the correspondence of Todd genus [4].

Lemm a 6.6. Let $p \geq 2$ be a prime integer and $k \geq 1, j \geq 1$ be integers. Then every Chern number of $C P\left(k p^{j}-1\right)$ is divisible by $p^{j}$.

Proof. It is easily seen by induction on $j \geq 1$ that $(1+x)^{p^{j}}=\left(1+x^{p}\right)^{p^{j-1}}+$ $p^{j} F_{(j)}(x)$ for some polynomial $F_{(j)}(x)$ with integer coefficients. Therefore the total Chern class of $C P\left(k p^{j}-1\right)$ is expressed as $(1+u)^{k p^{j}}=\left\{\left(1+u^{p}\right)^{p^{j-1}}+p^{j} F_{j}(u)\right\}^{k}$, 
where $u \in H^{2}\left(C P\left(k p^{j}-1\right) ; Z\right)$ is the first Chern class of $\eta$. So the Chern class $c_{i}\left(C P\left(k p^{j}-1\right)\right)$ is divisible by $p^{j}$ if $i$ is not a multiple of $p$. Since $k p^{j}-1$ is not a multiple of $p$, any monomial in $c_{1}, \cdots, c_{k p^{j}-1}$ of dimension $k p^{j}-1$ should contain some $c_{i}$ with $i$ not a multiple of $p$. Therefore the lemma follows.

Proposition 6.7 (cf. [2], [3], [5], [6], [7]). Let $p \geq 2, r \geq 1, a \geq 1$ be integers such that $p$ is prime and $(r, p)=1$. Put $m=p^{a} r$.

(1) All Chern numbers of $V_{i}^{(m)}(i \geq 0)$ are divisible by $m$.

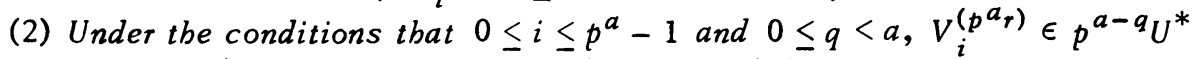
if and only if $p^{q-1} \nmid i+1$. In particular, $V_{0}^{(m)}=m, V_{p-1}^{(m)} \equiv p^{-1}\left(m-m^{p}\right)[C P(p-1)]$ $\bmod \left(\right.$ decomposables in $\left.U^{*}\right) \cap p^{2 a} U^{*}$ and $S_{\left(p^{j}-1\right)}\left[V_{p^{j}-w^{*}}^{(m)}+p^{-j} m^{p^{j}}\left[C P\left(p^{j}-1\right)\right]\right]=p^{a}$. So the classes $V_{p^{j}-1}^{(p)}$ are Milnor base elements for $U^{*} / p U^{*}$.

(3) In $U_{*}\left(L^{n}(m)\right)$ (and $\Omega_{*}\left(L^{n}(m)\right)$ when $m$ is odd) with $n \geq 1$, the order of the class $\left[L^{j}(m), i\right](0 \leq j<n)$ is $p^{d^{\prime}} m$ where $d$ is the integer determined by $2 d(p-1)<2 j+1<2(d+1)(p-1)$ and $r^{\prime}$ is the integer with $\left(r^{\prime}, p\right)=1$ which is determined analogously as $d$ from the prime decomposition of $r$. Moreover we have

$$
p^{d-1} r_{m}^{\prime}\left[L^{d(p-1)}(m), i\right]=\left(-1^{\prime d} p^{a-1} r r^{\prime}\left[C P^{(}(p-1)\right]^{d}\left[L^{0}(m), i\right] .\right.
$$

Proof. Let $g(X) \in U^{*} \otimes Q[[X]]$ be the logarithm of the formal group law $F(X, Y)$, i.e. $g(F(X, Y))=g(X)+g(Y)$. Miščenko's theorem [10, Appendix 1] asserts that $g(X)=\Sigma_{0 \leq k}([C P(k)] /(k+1)) X^{k+1}$. Comparing the coefficients of $X^{i}$ in the equation $\left(\Pi_{1 \leq j \leq i} j\right) g\left([m]_{F}(X)\right)=\left(\Pi_{1 \leq j \leq i} j\right)_{m} g(X)$, we obtain $V_{0}^{(m)}=m$ and, for $i \geq 2$,

$$
\left(\prod_{1 \leq j \leq i} j\right) V_{i-1}^{(m)}+G_{(i)}\left(V_{0}^{(m)}, \cdots, V_{i=2}^{(m)}\right)=\left(\prod_{1 \leq j \leq i-1} j\right)\left(m-m^{i}\right)[C P(i-1)],
$$

where $G_{(i)}(\ldots)$ is a polynomial in the $V_{0}^{(m)}, \ldots, V_{i-2}^{(m)}$ with coefficients in $U^{*}$ and each monomial in $G_{(i)}(\ldots)$ is an integer multiple of the form

$$
\left(\prod_{1 \leq j \leq i ; j \neq k \geq 2} j\right)\left[C P(k-1 !] V_{j}^{(m)} \cdots V_{j_{k}}^{(m)}\right.
$$

with $j_{1}+\cdots+j_{k}>0$. Part (1) and (2) easily tollow from the above equation and 6.6 by induction.

Now we prove part (3) by induction on $j(0 \leq j<n)$. It is easily derived from the reduced bordism spectral sequence $([2],[7])$ that the order of $\left[L^{0}(m), i\right]$ is $m$ as asserted.

Suppose the assertion is proved for $0, \ldots, j-1(j \geq 1)$. We prove the assertion for $j$ dividing cases.

(i) In case $p-1+j$. By 6.3(1) we have 


$$
\beta_{2, j+1}=m\left[L^{j}(m), i\right]+\cdots+V_{p-1}^{(m)}\left[L^{j-p+1}(m), i\right]+\cdots=0 .
$$

Multiplying $p^{d} r^{\prime}$, we obtain $p^{d} r^{\prime} m\left[L^{j}(m), i\right]=0$ by the induction hypothesis and part (2).

Let $\Delta: U_{*}\left(L^{n}(m)\right) \rightarrow U_{*-2}\left(L^{n}(m)\right)$ be the ordinary Smith homomorphism. Then by the induction hypothesis, $\Delta\left(p^{d-1} r^{\prime} m\left[L^{i}(m), i\right]\right)=p^{d-1} r^{\prime} m\left[L^{j-1}(m), i\right] \neq 0$ as desired.

In case $j=d(p-1)$ for some $d>1$. As in case (i), we have

$$
p^{d-1} r^{\prime} m\left[L^{d(p-1)}(m), i\right]+\cdots+p^{d-1} r^{\prime} V_{p-1}^{(m)}\left[L^{(d-1)(p-1)}(m), i\right]+\cdots=0
$$

But by the induction hypothes is and part (2), this reduces to

$$
p^{d-1} r^{\prime} m\left[L^{d(p-1)}(m), i\right]=-p^{d-2} r^{\prime} m[C P(p-1)]\left[L^{(d-1)(p-1)}(m), i\right] .
$$

The assertion follows directly from this.

This completes the proof of the theorem.

Remark 6.8. Since we now know the structure of $U_{*}\left(L^{n}(m)\right)$, the method of Conner-Floyd [2, Theorem 40.1], permits us to generalize a result in [3] and [18] concerning the fixed point sets of a weakly complex involution to the case of a semifree weakly complex periodic maps of arbitrary period whose fixed point set is a $k$-dimensional manifold (for some fixed $k$ ) with trivial normal bundle in the sense of $[2, \S 40]$. (See also Kasparov [19].)

7. An application to equivariant maps. Let $m, q \geq 2$ be integers. Consider the periodic map $T(m q): S^{2 n+1} \rightarrow S^{2 n+1}$ defined in the preceding section. Since $(T(m q))^{q}=T(m), T(m q)$ induces a free periodic map $\bar{T}(q): S^{2 n+1} / T(m)=L^{n}(m)$ $\rightarrow L^{n}(m)$ of period $q$ sending $\left[Z_{0}, \ldots, Z_{n}\right]$ to $\left[\lambda(m q) Z_{0}, \ldots, \lambda(m q) Z_{n}\right]$ where $\lambda(m q)=\exp (2 \pi i / m q)$.

Let $A(q): M^{2 k+1} \rightarrow M^{2 k+1}$ be a free differentiable periodic map of period $q$ on a $(2 k+1)$-dimensional $C^{\infty}$-manifold $M^{2 k+1}$. Suppose there exists an equivariant map $\tilde{f}:\left(L^{n}(m), \bar{T}(q)\right) \rightarrow\left(M^{2 k+1}, A(q)\right)$. Taking the orbit space, $\tilde{f}$ induces a map

$$
f: L^{n}(m) / \bar{T}(q)=L^{n}(m q) \rightarrow 11^{2 k+1} / A(q)
$$

and $\tilde{f}$ becomes a homomorphism of principal $Z_{q}$-bundles over $f$. Let $\gamma_{m q}, \gamma_{q}, \xi_{q}$ be the complex line bundles associated to the principal $Z_{m q^{-}}, Z_{q^{-}}, Z_{q^{-}}$-bundles $S^{2 n+1} \rightarrow S^{2 n+1} / T(m q), L^{n}(m) \rightarrow L^{n}(m) / \bar{T}(q), M^{2 k+1} \rightarrow M^{2 k+1} / A(q)$, respectively.

Lemma 7.1. $f^{*} \xi_{q} \cong\left(\gamma_{m q}\right)^{m}$. 
Proof. It is clear from the definition of an induced bundle and an associated bundle that $f^{*} \xi_{q}=\gamma_{q}=\left(\gamma_{m q}\right)^{m}$.

By the naturality of the cobordism Chern classes, it follows from 7.1 that $f^{*} c_{1}\left(\xi_{q}\right)=c_{1}\left(\left\{\gamma_{m q}\right\}^{m}\right)=[m]_{F}\left(c_{1}\left(\gamma_{m q}\right)\right)$. Since $M^{2 k+1} / A(q)$ is also a $(2 k+1)$-dimensional $C^{\infty}$-manifold, $c_{1}\left(\xi_{q}\right)^{k+1}=0$ in $U^{*}\left(M^{2 k+1} / A(q)\right)$ for the dimensional reasons. So we obtain the following.

Lemma 7.2. If $\left\{[m]_{F}\left(c_{1}\right)\right\}^{k+1} \neq 0$ in $U^{*}\left(L^{n}(m q)\right)$ where $c_{1}=c_{1}\left(\gamma_{m q}\right)$, then there does not exist an equivariant map $\left(L^{n}(m), \bar{T}(q)\right) \rightarrow\left(M^{2 k+1}, A(q)\right)$.

Theorem 7.3. Let $p \geq 2, r \geq 1$ be integers sucb that $p$ is prime and that $(r, p)=1$. And let $a \geq 1, k \geq 0$ be integers.

(i) $\left\{\left[p^{a} r\right]_{F}\left(c_{1}\right)\right\}^{k+1}=0$ in $U^{*}\left(L^{n}\left(p^{a+1} r\right)\right)$ implies $n \leq k p^{a}$.

(ii) If $r=1$ in (i), then the converse bolds, i.e. $n \leq k p^{a}$ implies $\left\{\left[p^{a}\right]_{F}\left(c_{1}\right)\right\}^{k+1}=0$ in $U^{*}\left(L^{n}\left(p^{a+1}\right)\right)$.

Theorem 7.4. Let $p \geq 2$ be a prime integer and $r, r^{\prime} \geq 1$ be integers sucb that $(p, r)=\left(p, r^{\prime}\right)=1$. Further let $a, b \geq 1$ and $k \geq 0$ be integers.

Suppose there exists an equivariant map

$$
f:\left(L^{n}\left(p^{a} r\right), \bar{T}\left(p^{b} r^{\prime}\right)\right) \rightarrow\left(M^{2 k+1}, A\left(p^{b} r^{\prime}\right)\right)
$$

for some smooth periodic map $A\left(p^{b} r^{\prime}\right)$ on a smooth $(2 k+1)$-manifold $M^{2 k+1}$. Then

(i) $n \leq k$ if $a k-b \leq-1$ and this is the best possible result.

(ii) $n \leq k p^{a}$ if $a k-b \geq 0$ (Munkbolm-Nakaoka).

Remark 7.5. This is a generalization of a result of Vick [15]. In the original manuscript of this paper, the statement of Theorem 7.4 was weaker than the present one. While revising this article, I was informed by M. Nakaoka that, replacing the formal group in cobordism theory in Lemma 7.2 by that of $K$-theory and applying a number theoretic argument of Munkholm, On the Borsuk-Ulam theorem for $Z_{p}$ a actions on $S^{2 n-1}$ and maps $S^{2 n-1} \rightarrow R^{m}$ (Osaka J. Math. 7 (1970), 423-441), he has obtained the result stated in Theorem 7.4 (ii). This inspired me to carry out the detailed calculation below in cobordism theory to obtain Theorem 7.3. I also learned from Nakaoka that $\left(M^{2 k+1}, A\left(p^{b}{ }_{r}^{\prime}\right)\right)$ need only be a continuous free periodic map on a finite $C W$-complex thanks to the theory of co-index.

The rest of this section is devoted to the proof of 7.3 and 7.4 .

For a partition $w=\left(i_{1}, i_{2}, \ldots, i_{q}\right)$ of nonnegative integers, let $r(i, w)$ be the occurrence of $i$ in $w$, and let 


$$
\left(\begin{array}{l}
j \\
\omega
\end{array}\right)=\left\{\begin{array}{l}
0 \text { if }|\omega|>j \\
j ! /\left\{\prod_{1 \leq i}(r(i, \omega) !)\right\}(j-|\omega|) ! \text { if }|\omega| \leq j
\end{array}\right.
$$

Define $\partial_{p}^{j} V_{i}^{\left(p^{a}\right)} \in U^{*} \otimes Q$ for $j \geq 0$ by

$$
\begin{aligned}
& \partial_{p}^{0} V_{i}^{\left(p^{a} r\right)}=(1 / p) V_{i}^{\left(p^{a+1} r\right)}-V_{i}^{\left(p^{a} r\right)}, \\
& \partial_{p}^{j} V_{i}^{\left(p^{a} r\right)}=\partial_{p}^{j-1} V_{i}^{\left(p^{a} r\right)}-\frac{[C P(j)]}{j+1} \sum_{|\omega|=j+1 ;\|\omega\|=i-j}\left(\begin{array}{c}
j+1 \\
\omega
\end{array}\right) V_{\omega}^{\left(p^{a+1} r\right)}
\end{aligned}
$$

where $V_{\omega}^{\left(p^{a+1} r\right)}=V_{i_{1}}^{\left(p^{a+1} r\right)} \ldots V_{i_{q}}^{\left(p^{a+1} r\right)}$.

Put $U_{i}^{\left(p^{\left.a_{r}\right)}\right.}=\partial_{p}^{i} V_{i}^{\left(p^{\left.a_{r}\right)}\right.}\left(=\partial_{p}^{i+1} V_{i}^{\left(p^{\left.a_{r}\right)}\right.}=\cdots\right)$. Let $\left(\partial_{p}^{j} V^{\left(p^{\left.a_{r}\right)}\right)}\right)_{\omega}$ and $U_{\omega}^{\left(p^{\left.a_{r}\right)}\right.}$

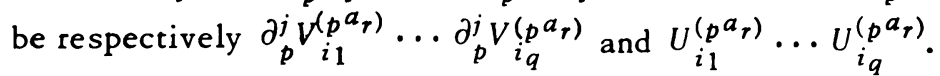

Lemma 7.6. In $U^{*}\left(L^{k p^{a+1}}\left(p^{a+1} r\right)\right)$, we have that

$$
s\left\{\left[p^{a} r\right]_{F}\left(c_{1}\right)\right\}^{k+1}=s p^{a} r\left(V_{p^{a}-1}^{\left(p^{a} r\right)}\right)_{1}^{k} c_{1}^{k p^{a}+1}+\sum_{|\omega|=k+1}\left(\begin{array}{c}
k+1 \\
\omega
\end{array}\right) s U_{\omega}^{\left(p^{a} r\right)} c_{1}\|\omega\|+k+1
$$

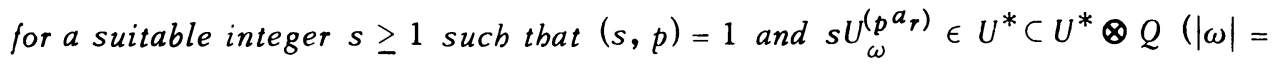
$\left.k+1,\|\omega\|+k+1 \leq k p^{a}+1\right)$.

Proof.

$$
\begin{aligned}
\left\{\left[p^{a} r\right]_{F}\left(c_{1}\right)\right\}^{k+1} & =\left\{\sum_{0 \leq j} V_{j}^{\left(p^{a} r\right)} c_{1}^{j+1}\right\}^{k+1}=\sum_{|\omega|=k+1}\left(\begin{array}{c}
k+1 \\
\omega
\end{array}\right) V_{\omega}^{\left(p^{a} r\right)} c_{1}^{\|\omega\|+k+1} \\
& =\sum_{0 \leq i \leq k+1}\left(\begin{array}{c}
k+1 \\
i
\end{array}\right)\left(p^{a} r^{i} \sum_{|\omega|=k+1-i ; \omega \neq 0} \sum_{\omega}^{k+1-i}\right) V_{\omega}^{\left(p^{a} r\right)} c_{1}\|\omega\|+k+1 .
\end{aligned}
$$

By 6.3, we have in $U^{*}\left(L^{k p^{a+1}}\left(p^{a+1} r\right)\right)$ that

$$
\left(p^{a+1} r\right)_{1}=-\left\{\sum_{1 \leq j} V_{j}^{\left(p^{a+1} r\right)} c_{1}^{j+1}\right\} \text {. }
$$

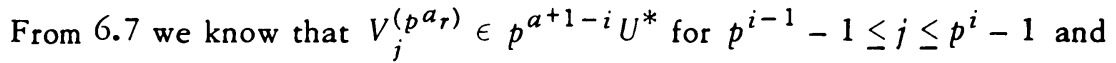
$0 \leq i \leq a$. So it is easily deduced that we can apply the formula (7.7) to

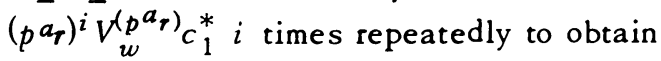

$$
\begin{aligned}
\left\{\left[p^{a} r\right]_{F}\left(c_{1}\right)\right\}^{k+1} & =\left\{\sum_{1 \leq i \leq k+1}\left(p^{a} r\right)\left(\frac{-1}{p}\right)^{i-1}\left(\begin{array}{c}
k+1 \\
i
\end{array}\right) \underset{p^{a}-1}{\left.V^{\left(p^{a+1} r\right)}\right)^{i-1}\left(V_{p^{a}-1}^{\left.\left(p^{a} r\right)\right) k+1-i}\right.}\right\} c_{1}^{k p^{a}+1} \\
& +\sum_{0 \leq i \leq k+1} \sum_{\omega, \omega^{\prime}}\left(\frac{-1}{p}\right)^{i}\left(\begin{array}{c}
k+1-i \\
\omega
\end{array}\right)\left(\begin{array}{c}
i \\
\omega^{\prime}
\end{array}\right) V_{\omega^{\prime}}^{\left(p^{a+1} r\right)} V_{\omega}^{\left(p^{a} r\right)} c_{1}^{\|\omega\|+\left\|\omega^{\prime}\right\|+k+1},
\end{aligned}
$$

the last summation being taken over all partitions $\omega$ and $\omega^{\prime}$ of positive integer components with $|\omega|=k+1-i$ and $\left|\omega^{\prime}\right|=i$. 
Notice that the order of $c_{1}^{k p^{a+1}}=D^{-1}\left[L^{0}\left(p^{a+1} r\right), i\right]$ is $p^{a+1} r, V_{i}^{\left(p^{a+1} r\right)}=$ $p\left(V_{i}^{\left(p^{a}\right)}-\partial_{p}^{0} V_{i}^{\left(p^{\left.a_{r}\right)}\right)}\right.$ and $\partial_{p}^{0} V_{p^{a}-1}^{\left(p^{a}\right)} \in p U^{*}$. So

$$
\begin{aligned}
& \sum_{1 \leq i \leq k+1}\left(p^{a} r\right)\left(\frac{-1}{p}\right)^{i-1}\left(\begin{array}{c}
k+1 \\
i
\end{array}\right)\left(\begin{array}{c}
\left.V_{p^{a}-1}^{\left(p^{a+1}\right.} r\right) i-1 \\
v^{a}-1 \\
\left(p^{a} r\right)
\end{array}\right)^{k+1-i} c_{1}^{k p^{a}+1} \\
& =p^{a} r\left(\left[-(x-1)^{k+1}-x^{k+1}\right]_{x=1}\right)\left(\underset{p^{a}-1}{V_{\left(p^{a} r\right)}^{k} c_{1}^{k p^{a}+1}}=p^{a} r \underset{p^{a}-1}{\left.V_{1}^{\left(p^{a} r\right)}\right)^{k} c_{1}^{k p^{a}+1} .}\right.
\end{aligned}
$$

Analogously,

$$
\begin{array}{r}
\sum_{\omega, \omega^{\prime}}\left(\frac{-1}{p}\right)^{i}\left(\begin{array}{c}
k+1-i \\
\omega
\end{array}\right)\left(\begin{array}{c}
i \\
\omega^{\prime}
\end{array}\right) V_{\omega^{\prime}}^{\left(p^{a+1} r\right)} V_{\omega}^{\left(p^{a} r\right)} c_{1}^{\|\omega\|+\left\|\omega^{\prime}\right\|+k+1} \\
=(-1)^{k+1} \sum_{\left|\omega^{\prime}\right|=k+1}\left(\begin{array}{c}
k+1 \\
\omega^{\prime}
\end{array}\right)\left(\partial_{p}^{0} V^{\left(p^{a} r\right)} \omega_{\omega^{\prime}} c_{1}^{\left\|\omega^{\prime}\right\|+k+1}\right.
\end{array}
$$

We carry out this kind of transformation of formulas inductively

$t_{j} s_{j} \sum_{|\omega|=k+1}\left(\begin{array}{c}k+1 \\ \omega\end{array}\right)\left(\partial_{p}^{j} V^{\left(p^{a} r\right)}{ }_{\omega^{c}} c_{1}^{\|\omega\|+k+1}=s_{j+1} \sum_{|\omega|=k+1}\left(\begin{array}{c}k+1 \\ \omega\end{array}\right)\left(\partial_{p}^{j+1} V^{\left(p^{a} r\right)} \omega_{\omega} c_{1}^{\|\omega\|+k+1}\right.\right.$

with $\left(t_{j}, p\right)=\left(s_{j}, p\right)=1, s_{j+1}=t_{j} s_{j}$ and $s_{j+1}\left(\partial_{p}^{j+1} V^{\left(p_{r}\right)_{\omega}} \in U^{*}\right.$.

So, after sufficiently many iterations, this formula stabilizes to

$$
s \sum_{|\omega|=k+1}\left(\begin{array}{c}
k+1 \\
\omega
\end{array}\right) U_{\omega}^{\left(p^{a} r\right)} c_{1}\|\omega\|+k+1
$$

This proves the lemma.

Proof of Theorem 7.3. Define $\partial^{j} U_{i}^{\left(p^{a} r\right)} \in U^{*} \otimes Q$ by

$$
\begin{aligned}
& \partial^{0} U_{i}^{\left(p^{a} r\right)}=U_{i}^{\left(p^{a} r\right)} \\
& \partial^{j} U_{i}^{\left(p^{a} r\right)}=\partial^{j-1} U_{i}^{\left(p^{a} r\right)}-\frac{[C P(j)]}{j+1} \sum_{|\omega|=j+1 ;\|\omega\|=i-j}\left(\begin{array}{c}
j+1 \\
\omega
\end{array}\right) V_{\omega}^{\left(p^{a} r\right)} \quad(j \geq 1),
\end{aligned}
$$

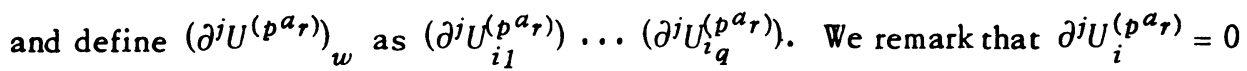
for $j$ greater than or equal to $i$ by virtue of Miščnko's theorem. (See the proof of $6.7(1),(2)$.

Analogously as in the proof of Lemma.7.6, we can find a suitable integer $s_{j}^{\prime} \geq 1$ for every $j \geq 1$ such that $\left(s_{j}^{\prime}, p\right)=1$ and that 


$$
\begin{aligned}
& \sum_{|\omega|=k+1}\left(\begin{array}{c}
k+1 \\
\omega
\end{array}\right) s_{j}^{\prime} U_{\omega}^{\left(p^{a}{ }^{2}\right)} c_{1}\|\omega\|+k+1 \\
&= \sum_{(k+1) /(j+1)_{s} \leq k+1}[M(j, m)]\left(\sum_{|\omega|=m}\left(\begin{array}{l}
m \\
\omega
\end{array}\right)\left(\partial^{j} U^{\left(p^{a} r\right)}\right)\right) c_{1}^{*} \\
&+\sum_{m^{\prime}>k+1}\left[N\left(j, m^{\prime}\right)\right]\left(\sum_{\left|\omega^{\prime}\right|=m^{\prime}}\left(\begin{array}{c}
m^{\prime} \\
\omega^{\prime}
\end{array}\right) U_{\omega^{\prime}}^{\left(p^{a} r\right)}\right) c_{1}^{*}
\end{aligned}
$$

where $[M(j, m)],\left[N\left(j, m^{\prime}\right)\right]$ are polynomials in $U^{*} \otimes Q$ such that $[M(j, m)]\left(\partial^{j} U_{w}^{\left(p^{a} r\right)}\right)$, $\left[N\left(j, m^{\prime}\right)\right] U_{w}^{\left(p^{a}{ }_{r}\right)} \in U^{*} \subset U^{*} \otimes Q$. Therefore, for sufficiently large $j$, we have

$$
\begin{aligned}
& s_{j}^{\prime} \sum_{|\omega|=k+1}\left(\begin{array}{c}
k-1 \\
\omega^{\prime}
\end{array}\right) U_{\omega}^{\left(p^{a} r\right)} c_{1}\|\omega\|+k+1 \\
& =\sum_{m^{\prime}>k+1}\left[N\left(j, m^{\prime}\right)\right]\left(\sum_{\left|\omega^{\prime}\right|=m^{\prime}}\left(\begin{array}{c}
m^{\prime} \\
\omega^{\prime}
\end{array}\right) U_{\omega^{\prime}}^{\left(p^{a} r\right)}\right) c_{1}^{*} \text {. }
\end{aligned}
$$

We can perform the same procedure for every $m^{\prime}$ in the right side of $(7.8)$ to obtain

$$
\sum_{m^{\prime \prime}>k+2}\left[N^{\prime}\left(j^{\prime}, m^{\prime}\right)\right]\left(\sum_{\left|\omega^{\prime \prime}\right|=m^{\prime \prime}}\left(\begin{array}{c}
m^{\prime \prime} \\
\omega^{\prime \prime}
\end{array}\right) U_{\omega^{\prime \prime}}^{\left(p^{a} r\right)}\right) c_{i^{\circ}}^{*}
$$

After sufficiently many iterations of this procedure, all partitions appearing in the right side of (7.8) are made to have their length as large as we please. So the right side of (7.8) becomes zero since $U_{0}^{\left(p^{a} r\right)}=0$ and $c_{1}^{i}=0$ for $i$ greater than $k p^{a}+1$. Consequently, for a suitable $s \geq 1$ with $(s, p)=1$, we have

$$
s\left\{\left[p^{a} r\right]_{F}\left(c_{1}\right)\right\}^{k+1}=s\left(p^{a} r\right)\left(\underset{p^{a}-1}{\left(p^{a} r\right)}\right)_{1}^{k} c_{1}^{k p^{a}+1}
$$

in $U^{*}\left(L^{k p^{a+1}}\left(p^{a+1} r\right)\right)$. Since $c_{1}^{k p^{a+1}}=D^{-1}\left[L^{0}\left(p^{a+1} r, i\right)\right]$, the theorem easily follows from (7.9) by virtue of 6.7(2), (3).

Proof of Theorem 7.4. (i) $\left\{\left[p^{a} r\right]_{F}\left(c_{1}\right)\right\}^{k+1}=\left(p^{a} r\right)^{k+1} c_{1}^{k+1}$ in $U^{*}\left(L^{k+1}\left(p^{a+b} r r^{\prime}\right)\right)$. By the hypothesis, $\left(p^{a} r\right)^{k+1} \nmid p^{a+b} r^{\prime}$ and so $\left\{\left[p^{a} r\right]_{F}\left(c_{1}\right)\right\}^{k+1} \neq 0$, which implies $n \leq k$ by 7.2 .

Conversely, if $n \leq k$, there exists the equivariant map $f:\left(L^{k}(m), \bar{T}(q)\right) \rightarrow$ $\left(s^{2 k+1}, T(q)\right)$ defined by

$$
f\left(\left[z_{0}, \cdots, z_{k}\right]\right)=\frac{1}{\sqrt{\left|z_{0}\right|^{2 m}+\cdots+\left|z_{k}\right|^{2 m}}}\left(z_{0}^{m}, \cdots, z_{k}^{m}\right) .
$$


(ii) It suffices to prove the case for $b=r^{\prime}=1$, which follows immediately from 7.2 and 7.3 .

\section{REFERENCES}

1. M. F. Atiyah, Bordism and cobordism, Proc. Cambridge Philos. Soc. 57 (1961), 200-208. MR 23 \#A4150.

2. P. E. Conner and E. E. Floyd, Differentiable periodic maps, Ergebnisse der Mathematik und ihrer Grenzgebiete, N. F., Band 33, Academic Press, New York; SpringerVerlag, Berlin, 1964. MR $31 \# 750$.

3. - Periodic maps which preserve a complex structure, Bull. Amer. Math. Soc. 70 (1964), 574-579. MR 29 \#1653.

4. - The relation of cobordism to $K$-theories, Lecture Notes in Math., no. 28, Springer-Verlag, Berlin and New York, 1966. MR 35 \#344.

5. T. tom Dieck, Bordism of G-manifolds and integrality theorems, Topology 9 (1970), 345-358. MR $42 \# 1148$.

6. C. H. Giffen, Weakly complex involutions and cobordism of projective spaces, Ann. of Math. (2) 90 (1969), 418-432. MR 40 \#6561.

7. M. Kamata, The structure of the bordism group $U_{*}\left(B Z_{p}\right)$, Osaka J. Math. 7 (1970), 409-416. MR 43 \#1203.

8. T. Kambe, The structure of $K_{\Lambda}$-rings of the lens space and their applications, J. Math. Soc. Japan 18 (1966), 135-146. MR 33 \#6646.

9. K. Komiya, Oriented bordism and involutions, Osaka J. Math. 9 (1972), 165-182.

10. S. P. Novikov, Methods of algebraic topology from the viewpoint of cobordism theories, Izv. Akad. Nauk SSSR Ser. Mat. 31 (1967), 855-951 = Math. USSR Izv. 1 (1967), 827-913. MR $36 \# 4561$.

11. R. E. Stong, Notes on cobordism theory, Princeton Univ. Press, Princeton, N. J.; Univ. of Tokyo Press, Tokyo, 1968. MR 40 \#2108.

12. - Bordism and involutions, Ann of Math. (2) 90 (1969), 47-74. MR 39 \#3503.

13. J. C. Su, A Note on the bordism algebra of involutions, Michigan Math. J . 12 (1965), 25-31. MR $31 \# 4040$.

14. F. Uchida, Bordism algebra of involutions, Proc. Japan Acad. 46 (1970), 615-619.

15. J. W. Vick, An application of $K$-theory to equivariant maps, Bull. Amer. Math. Soc. 75 (1969), 1017-1019. MR 39 \#6336.

16. C. T. C. Wall, Determination of the cobordism ring, Ann. of Math. (2) 72 (1960), 292-311. MR $22 \# 11403$.

17. C.-M. Wu, Bordism and maps of odd prime period, Osaka J. Math. 8(1971), 405-424.

18. T. Yoshida, On the K-theoretic characteristic numbers of weakly almost complex manifolds with involution (to appear).

19. G. G. Kasparov, Invariants of classical lens manifolds in cobordism theory, Izv. Akad. Nauk SSSR Ser. Mat. 33 (1969), 735-747 = Math. USSR Izv. 3 (1969), 695-705. MR $43 \# 1205$.

DEPARTMENT OF MATHEMATICS, OSAKA UNIVERSITY, OSAKA, JAPAN 